\title{
Localised module frames and Wannier bases from groupoid morita equivalences
}

Bourne, C.; Mesland, B.

\section{Citation}

Bourne, C., \& Mesland, B. (2021). Localised module frames and Wannier bases from groupoid morita equivalences. Journal of Fourier Analysis And Applications, 27(4).

doi:10.1007/s00041-021-09873-8

Version: $\quad$ Publisher's Version

License: $\quad$ Licensed under Article 25fa Copyright Act/Law (Amendment Taverne)

Downloaded from: $\quad$ https://hdl.handle.net/1887/3274970

Note: To cite this publication please use the final published version (if applicable). 


\title{
Localised Module Frames and Wannier Bases from Groupoid Morita Equivalences
}

\author{
Chris Bourne ${ }^{1,2}$ (D) Bram Mesland ${ }^{3}$ (D)
}

Received: 10 September 2020 / Revised: 5 May 2021 / Accepted: 24 June 2021 /

Published online: 4 August 2021

(c) The Author(s), under exclusive licence to Springer Science+Business Media, LLC, part of Springer Nature 2021

\begin{abstract}
Following the operator algebraic approach to Gabor analysis, we construct frames of translates for the Hilbert space localisation of the Morita equivalence bimodule arising from a groupoid equivalence between Hausdorff groupoids, where one of the groupoids is étale and with a compact unit space. For finitely generated and projective submodules, we show these frames are orthonormal bases if and only if the module is free. We then apply this result to the study of localised Wannier bases of spectral subspaces of Schrödinger operators with atomic potentials supported on (aperiodic) Delone sets. The noncommutative Chern numbers provide a topological obstruction to fast-decaying Wannier bases and we show this result is stable under deformations of the underlying Delone set.
\end{abstract}

Keywords Operator algebras · Groupoid and Morita equivalence · Gabor analysis · Wannier basis

Mathematics Subject Classification 46L08 · 46L55 · 81R60

\section{Introduction}

A key question in time-frequency analysis and related fields is the reconstruction of elements in a Hilbert space $\mathfrak{h}$ via a set of vectors $\left\{T_{j} w_{1}, \ldots, T_{j} w_{m}\right\}_{j \in J}$ spanning $\mathfrak{h}$ and where $\left\{T_{j}\right\}_{j \in J} \subset \mathcal{B}(\mathfrak{h})$ is some canonically defined set of operators. An important

\section{Chris Bourne}

chris.bourne@tohoku.ac.jp

Bram Mesland

b.mesland@math.leidenuniv.nl

1 WPI-AIMR, Tohoku University, 2-1-1 Katahira, Aoba-ku, Sendai 980-8577, Japan

2 RIKEN iTHEMS, 2-1 Hirosawa, Wako, Saitama 351-0198, Japan

3 Mathematisch Instituut, Universiteit Leiden, Niels Bohrweg 1, 2333 CA Leiden, The Netherlands 
example are (multi-window) Gabor frames, where given a locally compact and abelian group $G$, a frame on $L^{2}(G)$ is constructed via translation and modulation operators from a closed subset $\Lambda \subset G \times \widehat{G}$ on a window function. Morita equivalence bimodules of $C^{*}$-algebras, also called imprimitivity bimodules, have been shown to be a useful tool in the construction of such Gabor frames [1,2,27]. One may also consider frames generated from translations by elements of a discrete group or étale groupoid, which we call frames of translates, cf. [14, Chap. 9] or [8].

Closely related to frames of translates and arising from physics are Wannier bases. Given a Schrödinger-type operator $H$ with periodic potential acting on $L^{2}\left(\mathbb{R}^{d}, \mathbb{C}^{n}\right)$, a Wannier basis is an orthonormal basis of a spectral subspace of $H$ constructed from a finite set of functions along with their translations in $\mathbb{Z}^{d}$. Because the operator $H$ has a periodic potential, such bases exist by the Bloch-Floquet transform. The regularity of Wannier bases changes drastically depending on the topological properties of the spectral band of the Schrödinger operator, where a delocalised Wannier basis can be used as an indicator that the system has a non-trival topological phase, see $[15,16$, $24,29,32]$ for example. Wannier bases with exponential decay can be constructed for periodic and aperiodic Hamiltonians such that the compression of a position operator by the Fermi projection has uniform spectral gaps [40,41].

In the context of periodic systems with a space group translation symmetry $G \subset \mathbb{R}^{d}$, it was shown by Ludewig and Thiang that the existence of a fast-decaying Wannier basis is equivalent to whether a finitely generated and projective $C_{r}^{*}(G)$-module is free or not [26].

The purpose of this paper is twofold. First, using a similar framework to [2], we study the relation between frames of translates and Morita equivalence bimodules arising from groupoid equivalences. We then use this relation to extend the results of Ludewig and Thiang [26] on fast-decaying Wannier bases to étale groupoids. Regularity of frames is examined using pre-Morita equivalence bimodules of algebras defined from derivations and differential seminorms.

For both of our aims, our guiding example is the étale groupoid $\mathcal{G}_{\text {Del }}$ constructed from a Delone set $\Lambda \subset \mathbb{R}^{d}$ and which is equivalent to the crossed product groupoid of the translation action on the orbit space of $\Lambda, \Omega_{\Lambda} \rtimes \mathbb{R}^{d}$. In previous work [11,12], the index theory of $\mathcal{G}_{\mathrm{Del}}$ and its application to aperiodic topological phases was studied. In [23], Gabor frames of $L^{2}\left(\mathbb{R}^{d}\right)$ were constructed from Delone subsets of $\mathbb{R}^{2 d}$ with finite local complexity and the groupoid $\mathcal{G}_{\text {Del }}$ using results from [19]. Gabor duality was shown for Gabor frames constructed from model sets in [31]. We do not consider the important question of Gabor duality in this work.

\section{Outline and Main Results}

Because our results bring together constructions from time-frequency analysis, groupoids, $C^{*}$-modules and Morita equivalence, we give a brief overview of these concepts in Sect. 2. In Sect. 2.3, using the framework of differential seminorms (cf. [10]), we construct pre-Morita equivalence bimodules for pre- $C^{*}$-subalgebras obtained from a finite family of commuting unbounded $*$-derivations. 
In Sect. 3 we consider a groupoid equivalence $\mathcal{G} \leftarrow Z \rightarrow \mathcal{H}$ of Hausdorff, second countable and locally compact groupoids $\mathcal{G}$ and $\mathcal{H}$, where $\mathcal{H}$ is étale with a compact unit space $\mathcal{H}^{(0)}$. By choosing the evaluation state for some $x \in \mathcal{H}^{(0)}$ we obtain a translation action of the fibre $r^{-1}(x)$ on the Hilbert space localisation $\mathfrak{h}_{x}$ of the Morita equivalence bimodule between $C_{r}^{*}(\mathcal{G})$ and $C_{r}^{*}(\mathcal{H})$. This allows us to construct a normalised tight frame of translates for $\mathfrak{h}_{x}$ from the $C^{*}$-module frame of the Morita equivalence bimodule. Restricting to finitely generated and projective $C_{r}^{*}(\mathcal{H})$-submodules, we obtain a frame of translates with a finite generating set for a subspace of the Hilbert space localisation $\mathfrak{h}_{x}$. This frame is an orthonormal basis for all $x \in \mathcal{H}^{(0)}$ if and only if the finitely generated and projective module is free, and thus its class in the reduced $K$-theory of $C_{r}^{*}(\mathcal{H})$ is trivial. These results are extended in Sect. 4 to the case of abstract transversals with a normalised 2-cocycle twist.

We apply these results to the étale Delone groupoid in Sect. 5. We consider a magnetic Schrödinger operator with an atomic potential $v$ arranged on a Delone set $\Lambda \subset \mathbb{R}^{d}$

$$
H_{\Lambda}=\sum_{j=1}^{d}\left(-i \frac{\partial}{\partial x_{j}}-A_{j}\right)^{2}+\sum_{p \in \Lambda} v(\cdot-p)
$$

where $A$ is the magnetic potential. Results by Bellissard et al. show that for sufficiently regular $v, H_{\Lambda}$ and its magnetic translates are affiliated to the crossed product $C^{*}$-algebra $C_{r}^{*}\left(\Omega_{\Lambda} \rtimes \mathbb{R}^{d}, \theta\right)$ with $\theta$ a magnetic twist [5,6]. We show that for any Delone set $\mathcal{L}$ in the transversal subset $\Omega_{0} \subset \Omega_{\Lambda}$, a gapped spectral subspace of $H_{\mathcal{L}}$ has a normalised tight frame built from the magnetic translations $\left\{U_{y}\right\}_{y \in \mathcal{L}}$. This frame is an orthonormal basis if and only if the corresponding finitely generated and projective $C_{r}^{*}\left(\mathcal{G}_{\text {Del }}, \theta\right)$-module is free. Using derivations of the groupoid algebras and differential seminorms, we show this normalised tight frame has faster than polynomial decay. We therefore see that topological properties of spectral subspaces of the Delone Schrödinger operator can be related to the regularity of (aperiodic) Wannier bases.

The regularity of such Wannier basis is closely related to the Localisation Dichotomy Conjecture for non-periodic insulators raised in [29, Section 5 (arXiv version)] and further studied in $[25,30]$. We prove a weaker version of this conjecture in Sect. 5.3 and show that the existence of Wannier bases with faster than polynomial decay is equivalent to the existence of Wannier bases such that $\sum_{j}\left(1+|x|^{2}\right)\left|w_{j}(x)\right|^{2} \in$ $L^{1}\left(\mathbb{R}^{d}\right)$. This in turn is equivalent to the spectral projection defining a freely generated $C_{r}^{*}\left(\mathcal{G}_{\text {Del }}, \theta\right)$-module. Our results are weaker than those posed in [29] as we consider a family of Schrödinger operators and Hilbert spaces rather than a single Hamiltonian. Similarly we do not consider Wannier bases with exponential decay.

By relating the existence of a localised Wannier basis to a $K$-theoretic property, the noncommutative Chern numbers for $C_{r}^{*}\left(\Omega_{\Lambda} \rtimes \mathbb{R}^{d}, \theta\right)$ and $C_{r}^{*}\left(\mathcal{G}_{\text {Del }}, \theta\right)$ studied in [11-13] give a topological obstruction to a Wannier basis with fast decay. We also show that these Chern number formulas are continuous under deformations of the magnetic field or Delone atomic potential provided the spectral gap stays open. This 
implies that a non-localised Wannier basis is stable under deformations of the atomic potential (e.g. from a periodic lattice to an aperiodic point pattern).

\section{Preliminaries}

\subsection{Hilbert Space Frames}

Let us recall a few basic definitions from time-frequency analysis.

Definition 2.1 Let $\mathfrak{h}$ be a Hilbert space and $\left\{g_{j}\right\}_{j \in J}$ is a collection of elements in $\mathfrak{h}$. We say that $\left\{g_{j}\right\}_{j \in J}$ form a Hilbert space frame if there are constants $C, D>0$ such that

$$
C\|\psi\|^{2} \leq \sum_{j \in J}\left|\left\langle g_{j}, \psi\right\rangle\right|^{2} \leq D\|\psi\|^{2} \text { for all } \psi \in \mathfrak{h} \text {. }
$$

If $C=D$, then $\left\{g_{j}\right\}_{j \in J}$ is called a tight frame. If $C=D=1$, then $\left\{g_{j}\right\}_{j \in J}$ is called a normalised tight frame or Parseval frame.

Orthonormal bases are obvious examples of normalised tight frames. The restriction of an orthonormal basis of a Hilbert space to a closed subspace yields a normalised tight frame for the subspace. Normalised tight frames always arise as a compression of an orthonormal basis.

Proposition 2.2 [20, Sect. 1] Let $\left\{g_{j}\right\}_{j \in J}$ be a normalised tight frame of a Hilbert space $\mathfrak{h}_{1}$. Then there is Hilbert space $\mathfrak{h}_{2}$ and an orthonormal basis $\left\{e_{j}\right\}_{j \in J}$ of $\mathfrak{h}_{1} \oplus \mathfrak{h}_{2}$ such that $g_{j}=\operatorname{pr}_{1}\left(e_{j}\right)$.

Any Hilbert space frame yields an invertible frame operator

$$
S: \mathfrak{h} \rightarrow \mathfrak{h}, \quad S(\psi)=\sum_{j \in J} g_{j}\left\langle g_{j}, \psi\right\rangle
$$

We note that, in contrast to the Gabor analysis literature, we work with inner-products that are linear on the right. This is to make our results more easily compatible with right $C^{*}$-modules and their Hilbert space localisations (Definition 2.11 below). Because $S$ is invertible, one obtains a reconstruction formula for elements in $\mathfrak{h}$.

$$
\psi=\sum_{j \in J} S^{-1} g_{j}\left\langle g_{j}, \psi\right\rangle, \quad \psi \in \mathfrak{h}
$$

The elements $\left\{S^{-1} g_{j}\right\}_{j \in J}$ are called the dual frame to $\left\{g_{j}\right\}_{j \in J}$. 


\subsection{Pre- $C^{*}$-Modules and Morita Equivalence}

Following a similar framework to [2], we will use $C^{*}$-modules and Morita equivalence bimodules to study questions around frames of translates for their Hilbert space localisations. We now recall some basic definitions and establish notation. Further details can be found in $[9,35]$.

We will also be interested in the case where the $C^{*}$-algebras $A$ and $B$ have dense *-subalgebras $\mathcal{A}$ and $\mathcal{B}$.

Definition 2.3 Let $B$ be a $C^{*}$-algebra, $\mathcal{B} \subset B$ a dense $*$-subalgebra and $\mathcal{E}_{\mathcal{B}}$ a vector space that is also a right $\mathcal{B}$-module. We say that $\mathcal{E}_{\mathcal{B}}$ is a right inner product $\mathcal{B}$-module if there is a sesquilinear pairing $\left(e_{1}, e_{2}\right) \mapsto\left(e_{1} \mid e_{2}\right)_{\mathcal{B}} \in \mathcal{B}$ linear in the second variable such that for $e_{1}, e_{2}, e \in \mathcal{E}$

$$
\begin{array}{ll}
\left(e_{1} \mid e_{2}\right)_{\mathcal{B}}=\left(e_{2} \mid e_{1}\right)_{\mathcal{B}}^{*}, & \left(e_{1} \mid e_{2} \cdot b\right)_{\mathcal{B}}=\left(e_{1} \mid e_{2}\right)_{\mathcal{B}} b \\
(e \mid e)_{\mathcal{B}} \geq 0 \in B, & (e \mid e)_{\mathcal{B}}=0 \Rightarrow e=0 .
\end{array}
$$

An inner product module $\mathcal{E}_{\mathcal{B}}$ is called full if $\operatorname{span}\left\{\left(e_{1} \mid e_{2}\right): e_{1}, e_{2} \in \mathcal{E}_{\mathcal{B}}\right\}$ is $C^{*}$-norm dense in $B$. If $B$ is a $C^{*}$-algebra, a $C^{*}$-module is a right inner product $B$-module that is complete in the norm $\|e\|^{2}:=\left\|(e \mid e)_{B}\right\|_{B}$.

Given a $C^{*}$-algebra $A$ and dense $*$-subagebra $\mathcal{A}$, a left inner product $\mathcal{A}$-module $\mathcal{A}_{\mathcal{E}}$ can be analogously defined, where the sesquilinear pairing $\left(e_{1}, e_{2}\right) \mapsto \mathcal{A}_{\mathcal{A}}\left(e_{1} \mid e_{2}\right)$ is linear in the first variable and $\mathcal{A}\left(a \cdot e_{1} \mid e_{2}\right)=a_{\mathcal{A}}\left(e_{1} \mid e_{2}\right)$. In case the algebras $\mathcal{A}$ and $\mathcal{B}$ and left/right inner product module structure are clear from context, we will suppress subscripts and write $\mathcal{E}$ for ${ }_{\mathcal{A}} \mathcal{E}_{\mathcal{B}}$.

On an inner product module $\mathcal{E}_{\mathcal{B}}$, the norm $\|e\|^{2}:=\left\|(e \mid e)_{B}\right\|_{B}$ is defined and the completion of $\mathcal{E}_{\mathcal{B}}$ in this norm is a right $C^{*}$-module $E_{B}$ over $B$. For a right inner product $\mathcal{B}$-module, the $*$-algebra of finite rank operators $\operatorname{Fin}_{\mathcal{B}}\left(\mathcal{E}_{\mathcal{B}}\right)$ is defined to be the algebraic span of the finite-rank operators $\left\{\Theta_{e_{1}, e_{2}}^{R}\right\}_{e_{1}, e_{2} \in \mathcal{E}}$, where

$$
\Theta_{e_{1}, e_{2}}^{R}\left(e_{3}\right)=e_{1} \cdot\left(e_{2} \mid e\right)_{\mathcal{B}}, \quad\left(\Theta_{e_{1}, e_{2}}^{R}\right)^{*}:=\Theta_{e_{2}, e_{1}}^{R}, \quad e_{1}, e_{2}, e \in \mathcal{E} .
$$

When $E_{B}$ is a $C^{*}$-module over a $*$-algebra $B$, the compact endomorphisms $\mathbb{K}_{B}(E)$ are defined as the operator norm closure of $\operatorname{Fin}_{B}(E)$. It is a closed two-sided ideal in the $C^{*}$-algebra $\operatorname{End}_{B}^{*}(E)$ of adjointable operators on $E_{B}$.

Definition 2.4 Let $\mathcal{E}_{\mathcal{B}}$ be a right inner product $\mathcal{B}$-module. A set $\left\{e_{1}, \ldots, e_{n}\right\}$ is called a finite module frame if

$$
\operatorname{Id}_{\mathcal{E}}=\sum_{k=1}^{n} \Theta_{e_{k}, e_{k}}^{R}
$$

If $E_{B}$ is a right $C^{*}$-module, a countable subset $\left\{e_{j}\right\}_{j \in J} \subset E_{B}$ is a (right) $C^{*}$-module frame if $\sum_{j} \Theta_{e_{j}, e_{j}}^{R}$ converges strictly to $\operatorname{Id}_{E}$. 
We remark that any countably generated right $C^{*}$-module over a $\sigma$-unital algebra $B$ admits a $C^{*}$-module frame [9,35]. If an inner product module $\mathcal{E}_{\mathcal{B}}$ admits a finite frame $\left\{v_{j}\right\}_{j=1}^{n}$, then there is a projection $p=p^{*}=p^{2} \in M_{n}(\mathcal{B})$ and right module maps

$$
\begin{array}{ll}
S: \mathcal{E} \rightarrow \mathcal{B}^{n}, & R: \mathcal{B}^{n} \rightarrow \mathcal{E}, \\
S(e)=\left(\left(v_{j} \mid e\right)_{\mathcal{B}}\right)_{j=1}^{n}, & R\left(\left(b_{j}\right)_{j=1}^{n}\right)=\sum_{j=1}^{n} v_{j} \cdot b_{j},
\end{array}
$$

that restrict to isomorphisms $S: \mathcal{E} \rightarrow p \mathcal{B}^{n}$ and $R: p \mathcal{B}^{n} \rightarrow \mathcal{E}$. In particular

$$
p=\left(v_{i} \mid v_{j}\right)_{\mathcal{B}} \in M_{n}(\mathcal{B}), \quad R \circ S=\operatorname{Id}_{\mathcal{E}}, \quad e=R \circ S(e)=\sum_{j=1}^{n} v_{j} \cdot\left(v_{j} \mid e\right)_{\mathcal{B}}=\sum_{j=1}^{n} \Theta_{v_{j}, v_{j}}^{R}(e) .
$$

Similar formulas hold for left modules with a finite frame.

Definition 2.5 Let $A$ and $B$ be $C^{*}$-algebras. An $A-B$ Morita equivalence bimodule is a full right- $B C^{*}$-module and full left- $A C^{*}$-module ${ }_{A} E_{B}$ such that

$\left(a \cdot e_{1} \mid e_{2}\right)_{B}=\left(e_{1} \mid a^{*} \cdot e_{2}\right)_{B}, \quad{ }_{A}\left(e_{1} \mid e_{2} \cdot b\right)={ }_{A}\left(e_{1} \cdot b^{*} \mid e_{2}\right), \quad{ }_{A}\left(e_{1} \mid e_{2}\right) \cdot e_{3}=e_{1} \cdot\left(e_{2} \mid e_{3}\right)_{B}$

for all $a \in A, b \in B$ and $e_{1}, e_{2}, e_{3} \in E$. We say that $A$ and $B$ are Morita equivalent if there is a Morita equivalence bimodule ${ }_{A} E_{B}$.

To distinguish left and right inner products, for $e_{1}, e_{2} \in E$ we use the notation

$$
\Theta_{e_{1}, e_{2}}^{L}\left(e_{3}\right)={ }_{A}\left(e_{3} \mid e_{1}\right) \cdot e_{2}, \quad \Theta_{e_{1}, e_{2}}^{R}\left(e_{3}\right)=e_{1} \cdot\left(e_{2} \mid e_{3}\right)_{B} .
$$

A full right- $B C^{*}$-module is a Morita equivalence bimodule between $\mathbb{K}_{B}(E)$ and $B$ with the $\mathbb{K}_{B}(E)$-valued inner product $\mathbb{K}(E)\left(e_{1} \mid e_{2}\right)=\Theta_{e_{1}, e_{2}}^{R}$. Hence $A$ is Morita equivalent to $B$ if and only if there is a full right- $B C^{*}$-module $E_{B}$ and a $*$-isomorphism $\phi: A \rightarrow \mathbb{K}_{B}(E)$.

Definition 2.6 Let $\mathcal{A}$ and $\mathcal{B}$ be dense $*$-subalgebras of $C^{*}$-algebras $A$ and $B$. A preMorita equivalence bimodule is an $\mathcal{A}-\mathcal{B}$ bimodule ${ }_{\mathcal{A}} \mathcal{E}_{\mathcal{B}}$, equipped with a full left- $\mathcal{A}$ valued and a full right- $\mathcal{B}$ valued inner product such that for any $a \in \mathcal{A}, b \in \mathcal{B}$ and $e, e_{1}, e_{2}, e_{3} \in \mathcal{E}$ the compatibility conditions

$\mathcal{A}_{\mathcal{A}}(e \cdot b \mid e \cdot b) \leq\|b\|^{2}{ }_{\mathcal{A}}(e \mid e), \quad(a \cdot e \mid a \cdot e)_{\mathcal{B}} \leq\|a\|^{2}(e \mid e)_{\mathcal{B}}, \quad \mathcal{A}_{\mathcal{A}}\left(e_{1} \mid e_{2}\right) \cdot e_{3}=e_{1} \cdot\left(e_{2} \mid e_{3}\right)_{\mathcal{B}}$,

are satisfied. Here $\|\cdot\|$ denotes the $C^{*}$-norm on the algebras $\mathcal{A}$ and $\mathcal{B}$, and the inequalities are in the $C^{*}$-algebras $A$ and $B$. 
As expected, a pre-Morita equivalence bimodule ${ }_{\mathcal{A}} \mathcal{E}_{\mathcal{B}}$ can be completed to a Morita equivalence bimodule ${ }_{A} E_{B}$, using either of the norms $\|e\|^{2}:=\left\|(e \mid e)_{\mathcal{B}}\right\|_{B}$ or $\|e\|^{2}:=\left\|_{\mathcal{A}}(e \mid e)\right\|_{A}$ see $[35$, Sect. 3.1]. We proceed with some definitions and results concerning frames in dense submodules of $C^{*}$-modules. For this we need our dense $*$-subalgebras to be equipped with additional analytic structure.

We provide a definition of smooth subalgebra of a $C^{*}$-algebra (see for instance [18, Definitions 3.25 and 3.26]) that is flexible enough for our purposes.

Definition 2.7 We say that a $*$-algebra $\mathcal{A}$ is a pre- $C^{*}$-algebra if it is

(i) Fréchet, i.e. complete and metrizable such that the multiplication is jointly continuous;

(ii) Isomorphic to a proper dense $*$-subalgebra $\iota(\mathcal{A})$ of a $C^{*}$-algebra $A$, where $\iota$ : $\mathcal{A} \hookrightarrow A$ is the inclusion map, and $\iota(\mathcal{A})$ is stable under the holomorphic functional calculus. That is, if $f$ is a holomorphic function on a neighbourhood of the spectrum of $a \in \iota(\mathcal{A})$, then $f(a) \in \iota(\mathcal{A})$.

Stability under the holomorphic functional calculus extends to nonunital algebras, since the spectrum of an element in a nonunital algebra is defined to be the spectrum of this element in the one-point unitization, though we must restrict to functions satisfying $f(0)=0$. Similarly, the definition of a Fréchet algebra does not require a unit.

The $K$-theory groups $K_{0}(\mathcal{A})$ and $K_{1}(\mathcal{A})$ can be defined for a pre- $C^{*}$-algebra $\mathcal{A}$, see [18, Sect. 3.8] or [9] for example. A useful feature of pre- $C^{*}$-algebras is that they contain all the $K$-theoretic information of their $C^{*}$-completion.

Proposition 2.8 [37] If $\mathcal{A}$ is a pre- $C^{*}$-algebra with $C^{*}$-completion $A$, then the map induced by the inclusion $\iota_{*}: K_{j}(\mathcal{A}) \rightarrow K_{j}(A)$ is an isomorphism for $j=0,1$.

Lemma 2.9 Let $\mathcal{A}$ and $\mathcal{B}$ be pre- $C^{*}$-algebras with $\mathcal{B}$ unital and ${ }_{\mathcal{A}} \mathcal{E}_{\mathcal{B}}$ a pre-Morita equivalence bimodule.

1. There is a finite left module frame $\left\{g_{1}, \ldots, g_{n}\right\} \subset \mathcal{E}$ and $1_{\mathcal{B}}=\sum_{k=1}^{n}\left(g_{k} \mid g_{k}\right)_{\mathcal{B}}$.

2. For any $p=p^{*}=p^{2} \in M_{n}(\mathcal{A}), p \mathcal{E}^{\oplus n}$ is a finitely generated and projective $\mathcal{B}$-module and there exists a finite right module frame $\left\{v_{1}, \ldots, v_{m}\right\} \subset p \mathcal{E}^{\oplus n}$.

Proof We write $A$ and $B$ for the $C^{*}$-algebra closures of $\mathcal{A}$ and $\mathcal{B}$ and $E$ for the $C^{*}$ module closure of $\mathcal{E}$, which is a Morita equivalence bimodule for the $C^{*}$-algebras $A$ and $B$.

(1) The $*$-algebra of finite rank operators $\operatorname{span}\left\{\Theta_{e_{1}, e_{2}}^{L}\right\}_{e_{1}, e_{2} \in \mathcal{E}}$ is $C^{*}$-norm dense in ${ }_{A} \mathbb{K}(E)$. Thus for $\varepsilon<1$ we can find $\left\{e_{1}, \ldots, e_{n}\right\} \subset \mathcal{E}$ such that the operator

$$
g:=\sum_{k=1}^{n} \Theta_{e_{k}, e_{k}}^{L}=\sum_{k=1}^{n}\left(e_{k} \mid e_{k}\right)_{\mathcal{B}} \in \mathcal{B},
$$

satisfies $\left\|1_{B}-g\right\|_{B}<\varepsilon$. Hence the positive element $g$ is invertible in $B$. Because the spectrum of $g$ is contained in the region of analycity of $f(z)=z^{-1}$ and $\mathcal{B}$ is stable under the holomorphic functional calculus, $g^{-1}$ and $g^{-1 / 2} \in \mathcal{B}$. Define $g_{k}:=e_{k} \cdot g^{-\frac{1}{2}} \in \mathcal{E}$, for $1, \ldots, n$ so that 


$$
\begin{aligned}
\sum_{k=1}^{n}\left(g_{k} \mid g_{k}\right) \mathcal{B} & =\sum_{k=1}^{n} \Theta_{g_{k}, g_{k}}^{L}=\sum_{k=1}^{n} \Theta_{e_{k} g^{-\frac{1}{2}}, e_{k} g^{-\frac{1}{2}}}^{L} \\
& =g^{-\frac{1}{2}}\left(\sum_{k=1}^{n} \Theta_{e_{k}, e_{k}}^{L}\right) g^{-\frac{1}{2}}=g^{-\frac{1}{2}} g g^{-\frac{1}{2}}=1_{\mathcal{B}},
\end{aligned}
$$

which proves the claim.

(2) Since $p$ is a compact operator on $E^{\oplus n}$, it is finite rank by [18, Corollary 3.10], so the module $W:=p E^{\oplus n}$ is finitely generated and projective over $B$. Write $\mathcal{W}:=p \mathcal{E}^{\oplus n}$ for the dense $\mathcal{B}$-submodule defined by $p \in M_{n}(\mathcal{A})$.

The *-algebra of finite rank operators $\operatorname{span}\left\{\Theta_{e_{1}, e_{2}}^{R}\right\}_{e_{1}, e_{2} \in \mathcal{W}}$ is $C^{*}$-norm dense in $\mathbb{K}_{B}(W)$. Hence for $\varepsilon<1$ there exist $w_{1}, \ldots, w_{m} \in \mathcal{W}$ such that $w:=\sum \Theta_{w_{k}, w_{k}}^{R}$

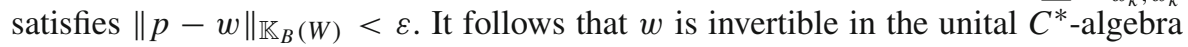
$\mathbb{K}_{B}(W) \cong p M_{n}(A) p$ and has spectrum contained in $B(1 ; \varepsilon)$. The spectrum of $w$ in $M_{n}(A)$ is thus contained in the disconnected set $B(1 ; \varepsilon) \cup\{0\}$. By spectral invariance, the same holds for the spectrum of $w \in M_{n}(\mathcal{A})$. Thus there is a function $f$, holomorphic on a neighborhood of the spectrum of $w$ such that $f(0)=0$ and $f(z)=z^{-\frac{1}{2}}$ for $z \in B(1 ; \varepsilon)$. Hence $f(w) \in M_{n}(\mathcal{A}) \cap p M_{n}(A) p=p M_{n}(\mathcal{A}) p$ and satisfies $f(w) w f(w)=p$. Now put $v_{k}:=f(w) w_{k}$ so that, as above,

$$
\sum_{k=1}^{m} \Theta_{v_{k}, v_{k}}^{R}=f(w)\left(\sum_{k=1}^{m} \Theta_{w_{k}, w_{k}}^{R}\right) f(w)=p,
$$

which proves the claim.

\subsection{Derivations, Pre-Morita Equivalence Bimodules and Localisation}

We now describe a general method to construct pre-Morita equivalence bimodules and pre- $C^{*}$-algebras from a family of densely defined derivations on a given $C^{*}$-algebra. For instance, the algebra $C^{\infty}(M)$, with $M$ a compact manifold, can be constructed in this way.

As a technical tool we will use the notion of differential seminorms introduced in [10, Definition 3.1]. The space $\ell^{1}(\mathbb{N})$ is an algebra in the convolution product $f * g(n):=\sum_{k \leq n} f(k) g(n-k)$. The subspace $\ell_{+}^{1}(\mathbb{N}):=\ell^{1}\left(\mathbb{N}, \mathbb{R}_{+}\right) \subset \ell^{1}(\mathbb{N})$ inherits the coordinatewise ordering from $\mathbb{R}_{+}$and satisfies $\ell_{+}^{1}(\mathbb{N}) \ell_{+}^{1}(\mathbb{N}) \subset \ell_{+}^{1}(\mathbb{N})$. Following [10], a differential seminorm on a subalgebra $\mathfrak{A} \subset A$ is a map $T: \mathfrak{A} \rightarrow \ell_{+}^{1}(\mathbb{N})$ such that $T(a)(0) \leq C\|a\|$ and $T(\lambda a)=|\lambda| T(a)$ and $T(a b) \leq T(a) * T(b)$. The functional $\int: \ell^{1}(\mathbb{N}) \rightarrow \mathbb{C}, f \mapsto \sum_{k \in \mathbb{N}} f(k)$, is positive and multiplicative, and if $T: \mathfrak{A} \rightarrow \ell_{+}^{1}(\mathbb{N})$ is a differential seminorm, then $\int T: \mathfrak{A} \rightarrow \mathbb{R}_{+}$is a submulitiplicative seminorm.

Proposition 2.10 (Cf. [10]) Let A be a $C^{*}$-algebra with norm $\|\cdot\|, \mathfrak{A} \subset A$ a dense $*$-subalgebra and $\left\{\partial_{j}: \mathfrak{A} \rightarrow \mathfrak{A}\right\}_{j=1}^{d}$ a finite family of commuting $*$-derivations. Then 
for $\alpha \in \mathbb{N}^{d}$ a multi-index,

$$
\|a\|_{n}=\sum_{|\alpha| \leq n} \frac{\left\|\partial^{\alpha} a\right\|}{\alpha !}, \quad \partial^{\alpha}=\partial_{1}^{\alpha_{1}} \ldots \partial_{d}^{\alpha_{d}}, \quad|\alpha|:=\sum_{k=1}^{n} \alpha_{k}, \quad \alpha !:=\prod_{k=1}^{d}\left(\alpha_{k} !\right),
$$

is a sequence of submutliplicative seminorms on $\mathfrak{A}$. Let $\mathcal{A}_{n}$ denote the closure of $\mathfrak{A}$ in the seminorms $\|\cdot\|_{k}, k \leq n$ and $\mathcal{A}_{\infty}:=\lim \mathcal{A}_{n}$ the Fréchet closure of $\mathfrak{A}$ in all the seminorms $\|\cdot\|_{n}$. Then for $n=0, \ldots, \infty, \overleftarrow{\mathcal{A}_{n}}$ is a pre-C $C^{*}$-algebra.

Proof Defining $\left(\begin{array}{l}\alpha \\ \beta\end{array}\right):=\prod_{k=1}^{d}\left(\begin{array}{l}\alpha_{k} \\ \beta_{k}\end{array}\right)$, one proves that $\partial^{\alpha}(a b)=\sum_{\beta \leq \alpha}\left(\begin{array}{l}\alpha \\ \beta\end{array}\right) \partial^{\beta}(a)$ $\partial^{\alpha-\beta}(b)$, by induction on $\alpha$. Following [10], for $n \in \mathbb{N}$ we consider the maps $T_{n}: \mathfrak{A} \rightarrow \ell_{+}^{1}(\mathbb{N}), \quad T_{n}(a)(k):=\frac{\sum_{|\alpha|=k}\left\|\partial^{\alpha} a\right\|}{\alpha !}$ for $k \leq n, \quad T_{n}(a)(k)=0$ for $k>n$.

Indeed the $T_{n}$ satisfy $T_{n}(a)(0)=\|a\|$ and $T_{n}(\lambda a)=|\lambda| T_{n}(a)$ as well as

$$
\begin{aligned}
T_{n}(a b)(k) & =\sum_{|\alpha|=k} \frac{1}{\alpha !}\left\|\partial^{\alpha}(a b)\right\| \leq \sum_{|\alpha|=k} \sum_{\beta \leq \alpha} \frac{1}{\beta !}\left\|\partial^{\beta}(a)\right\| \frac{1}{(\alpha-\beta) !}\left\|\partial^{\alpha-\beta}(b)\right\| \\
& \leq \sum_{|\alpha|+|\beta|=k} \frac{1}{\beta !}\left\|\partial^{\beta}(a)\right\| \frac{1}{\alpha !}\left\|\partial^{\alpha}(b)\right\| \leq T_{n}(a) * T_{n}(b)(k),
\end{aligned}
$$

which shows that $T_{n}$ is a differential algebra norm. Moreover for $k \geq 1$

$$
\begin{aligned}
T_{n}(a b)(k) \leq & \sum_{|\alpha|+|\beta|=k} \frac{1}{\beta !}\left\|\partial^{\beta}(a)\right\| \frac{1}{\alpha !}\left\|\partial^{\alpha}(b)\right\| \\
= & \|a\|\left(\sum_{|\beta|=k} \frac{1}{\beta !}\left\|\partial^{\beta}(b)\right\|\right)+\left(\sum_{|\alpha|=k} \frac{1}{\alpha !}\left\|\partial^{\alpha}(a)\right\|\right)\|b\| \\
& +\sum_{|\alpha|,|\beta| \geq 1} \frac{1}{\beta !}\left\|\partial^{\beta}(a)\right\| \frac{1}{\alpha !}\left\|\partial^{\alpha}(b)\right\|,
\end{aligned}
$$

so that each $T_{n}$ is of logarthmic order $\leq 1$ (see [10, Definition 3.4]). We then have $\|a\|_{n}=\int T_{n}(a)$ and the result for $\mathcal{A}_{n}$ follows from [10, Propositions 3.3 and 3.12]. The result for $\mathcal{A}_{\infty}$ then follows since $\mathcal{A}_{\infty}=\bigcap_{n=0}^{\infty} \mathcal{A}_{n}$ and the result holds for each $\mathcal{A}_{n}$.

Now let $\mathfrak{A} \subset A$ and $\mathfrak{B} \subset B$ be dense $*$-subalgebras and $\mathfrak{E}$ an $\mathfrak{A}-\mathfrak{B}$ pre-Morita equivalence bimodule. Suppose we are given a families of commuting $*$-derivations

$$
\left\{\partial_{j}^{L}\right\}_{j=1}^{d}: \mathfrak{A} \rightarrow \mathfrak{A}, \quad\left\{\partial_{j}^{R}\right\}_{j=1}^{d}: \mathfrak{B} \rightarrow \mathfrak{B},
$$


as well as a commuting family of operators $\nabla_{j}: \mathfrak{E} \rightarrow \mathfrak{E}$ such that for each $j$ and all $a \in C_{c}(\mathcal{G}), b \in C_{c}(\mathcal{H}), \xi, \eta \in C_{c}(Z)$ and $b \in \mathfrak{B}$ we have

$$
\begin{aligned}
\nabla_{j}(a \cdot \xi \cdot b) & =\partial_{j}^{L}(a) \cdot \xi \cdot b+a \cdot \nabla_{j}(\xi) \cdot b+a \cdot \xi \cdot \partial_{j}^{R}(b), \\
\partial_{j}^{R}(\xi \mid \eta)_{\mathfrak{B}} & =\left(\xi \mid \nabla_{j}(\eta)\right)_{\mathfrak{B}}-\left(\nabla_{j}(\xi) \mid \eta\right)_{\mathfrak{B}} .
\end{aligned}
$$

It is worth noting that the identity

$$
\partial_{j}^{L} \mathfrak{A}(\xi \mid \eta)=\mathfrak{A}\left(\xi \mid \nabla_{j}(\eta)\right)-\mathfrak{A}\left(\nabla_{j}(\xi) \mid \eta\right)
$$

is satisfied as well. By using Eqs. (3), (4) and the compatibility of left and right inner products, we find for $\xi, \eta, e \in \mathcal{E}$ :

$$
\begin{aligned}
\mathfrak{A}\left(\xi \mid \nabla_{j}(\eta)\right) \cdot e-\mathfrak{A}\left(\nabla_{j}(\xi) \mid \eta\right) \cdot e= & \xi \cdot\left(\nabla_{j}(\eta) \mid e\right)_{\mathfrak{B}}-\nabla_{j}(\xi) \cdot(\eta \mid e)_{\mathfrak{B}} \\
= & \xi \cdot\left(\eta \mid \nabla_{j}(e)\right)_{\mathfrak{B}}-\xi \cdot \partial_{j}^{R}(\eta \mid e) \\
& -\nabla_{j}(\xi) \cdot(\eta \mid e)_{\mathfrak{B}} \\
= & \xi \cdot\left(\eta \mid \nabla_{j}(e)\right)_{\mathfrak{B}}-\nabla_{j}\left(\xi \cdot(\eta \mid e)_{\mathfrak{B}}\right) \\
= & \left.\mathfrak{A}(\xi \mid \eta) \cdot \nabla_{j}(e)-\nabla_{j}(\mathfrak{A}(\xi \mid \eta) \cdot e)\right) \\
= & \partial_{j}^{L} \mathfrak{A}(\xi \mid \eta) \cdot e,
\end{aligned}
$$

so that (5) follows.

We write $\mathcal{A}_{n}, \mathcal{B}_{n}$ for the pre- $C^{*}$-algebra completions obtained through Proposition 2.10. Similarly we write

$$
\nabla^{\alpha}:=\nabla_{1}^{\alpha_{1}} \cdots \nabla_{d}^{\alpha_{d}}, \quad \alpha \in \mathbb{N}^{d}, \quad\|e\|_{n}:=\sum_{|\alpha| \leq n} \frac{\left\|\nabla^{\alpha}(e)\right\|}{\alpha !}
$$

as well as $\mathcal{E}_{n}$ for the completion of $\mathfrak{E}$ in the seminorms up to degree $n$, and $\mathcal{E}=\mathcal{E}_{\infty}$ for the completion of $\mathfrak{E}$ in all these seminorms.

Definition 2.11 Suppose that $E_{B}$ is a right $C^{*}$-module with $B$ a unital $C^{*}$-algebra and $\omega_{B}: B \rightarrow \mathbb{C}$ a state. The localisation $\mathfrak{h}_{\omega}$ is the Hilbert space that comes from the completion of $E$ in the inner product

$$
\left\langle e_{1}, e_{2}\right\rangle_{\omega}=\omega_{B}\left(\left(e_{1} \mid e_{2}\right)_{B}\right) .
$$

Remark 2.12 If ${ }_{A} E_{B}$ is a Morita equivalence bimodule and the state $\tau: B \rightarrow \mathbb{C}$ is a trace, then there is a canonical dual tracial weight $\operatorname{Tr}_{\tau}$ on $\mathbb{K}_{B}(E) \cong A$ such that $\operatorname{Tr}_{\tau}\left(\Theta_{e_{1}, e_{2}}^{R}\right)=\tau\left(\left(e_{2} \mid e_{1}\right)_{B}\right)$ for any $e_{1}, e_{2} \in E$. We can localise ${ }_{A} E_{B}$ with a left-linear inner-product from $\operatorname{Tr}_{\tau}$ to obtain the dual localisation space $\mathfrak{h}_{\omega}^{*}$. Hence in this special case, the localisation Hilbert space directly agrees with the Gabor analysis literature. See [2] for more details. 
Given any state $\omega: B \rightarrow \mathbb{C}$, the seminorms $\|\cdot\|_{n}$ induce a family of seminorms $\|\cdot\|_{n, \omega}$ on the Hilbert space localisation $\mathfrak{h}_{\omega}$,

$$
\|\xi\|_{n, \omega}:=\sum_{|\alpha| \leq n} \frac{\left\|\nabla^{\alpha}(\xi)\right\|_{\mathfrak{h}_{\omega}}}{\alpha !}, \quad n \in \mathbb{N} .
$$

Proposition 2.13 For $n=0, \ldots, \infty$ the space $\mathcal{E}_{n}$ is a $\mathcal{A}_{n}-\mathcal{B}_{n}$ pre-Morita equivalence bimodule. Moreover, for any state $\omega: B \rightarrow \mathbb{C}$, the image of $\mathcal{E}_{n}$ in $\mathfrak{h}_{\omega}$ is bounded in the seminorms $\|\cdot\|_{k, \omega}$ for $0 \leq k \leq n$.

Proof The space

$$
\mathcal{L}(\mathfrak{E}):=\left\{\left(\begin{array}{cc}
a & \xi \\
\eta^{*} & b
\end{array}\right): a \in \mathfrak{A}, \quad b \in \mathfrak{B}, \quad \xi, \eta \in \mathfrak{E}\right\}
$$

with multiplication and involution

$$
\begin{aligned}
\left(\begin{array}{ll}
a_{1} & \xi_{1} \\
\eta_{1}^{*} & b_{1}
\end{array}\right) \cdot\left(\begin{array}{ll}
a_{2} & \xi_{2} \\
\eta_{2}^{*} & b_{2}
\end{array}\right) & :=\left(\begin{array}{cc}
a_{1} a_{2}+\mathfrak{A}\left(\xi_{1} \mid \eta_{2}\right) & a_{1} \xi_{2}+\xi_{1} b_{2} \\
\left(a_{2}^{*} \eta_{1}\right)^{*}+\left(\eta_{2} b_{2}\right)^{*} & \left(\eta_{1} \mid \xi_{2}\right)_{\mathfrak{B}}+b_{1} b_{2}
\end{array}\right), \quad\left(\begin{array}{cc}
a & \xi \\
\eta^{*} & b
\end{array}\right)^{*} \\
& :=\left(\begin{array}{cc}
a^{*} & \eta^{*} \\
\xi & b^{*}
\end{array}\right)
\end{aligned}
$$

is an associative $*$-algebra, the linking algebra of $\mathfrak{E}$. It is a dense $*$-subalgebra of the linking $C^{*}$-algebra $\mathcal{L}(E)$ of the $C^{*}$-module closure $E$ of $\mathfrak{E}$. Using the identities (3), (4) and (5), one shows that the maps $\nabla_{j}$ induce a family of commuting $*$-derivations on the linking algebra via

$$
\Delta_{j}\left(\begin{array}{cc}
a & \xi \\
\eta^{*} & b
\end{array}\right):=\left(\begin{array}{cc}
\partial_{j}^{L} a & \nabla_{j}(\xi) \\
-\nabla_{j}(\eta)^{*} & \partial_{j}^{R} b
\end{array}\right)
$$

and the norms $\|e\|_{n}$ defined above are the restrictions of the norms obtained from the derivations $\Delta_{j}$. This proves that $\mathcal{E}_{n}$ is an $\mathcal{A}_{n}-\mathcal{B}_{n}$ bimodule. Since $\mathcal{E}_{n}$ is a subspace of $E$, the properties of Definition 2.6 will follow once we show that the left and right inner products on ${ }_{A} E_{B}$ take values in $\mathcal{A}_{n}$ and $\mathcal{B}_{n}$, respectively, when restricted to $\mathcal{E}_{n}$. This in turn follows since the inner products are realised as multiplications in the linking algebra. Lastly we have $\|e\|_{k, \omega} \leq\|e\|_{k}$ for any state $\omega$, so the image of $\mathcal{E}_{n}$ is bounded in each of the seminorms $\|e\|_{k, \omega}$ with $k \leq n$.

Remark 2.14 The pre-Morita equivalence bimodule $\mathcal{A}_{\infty} \mathcal{E}_{\infty} \mathcal{B}_{\infty}$ is the 'smoothest' bimodule over pre- $C^{*}$-algebras that we can consider from the derivations $\left\{\partial_{j}^{L}\right\}_{j=1}^{d}$ and $\left\{\partial_{j}^{R}\right\}_{j=1}^{d}$. The lower-order pre-Morita equivalence bimodules $\mathcal{A}_{n} \mathcal{E}_{n} \mathcal{B}_{n}$ for $1 \leq n<\infty$ will allow us to consider $C^{*}$-module and Hilbert space frames of differing regularity. This will be of use to us when considering the localisation dichotomy of Wannier bases in Sect. 5.3. 


\subsection{Groupoid C*-Algebras and Equivalence}

Groupoids provide a useful generalisation of groups and group actions on spaces. A standard reference for groupoid $C^{*}$-algebras is [36].

Definition 2.15 A groupoid is a set $\mathcal{G}$ with a subset $\mathcal{G}^{(2)} \subset \mathcal{G} \times \mathcal{G}$, a multiplication map $\mathcal{G}^{(2)} \rightarrow \mathcal{G},(\gamma, \xi) \mapsto \gamma \xi$ and an inverse $\mathcal{G} \rightarrow \mathcal{G}, \gamma \mapsto \gamma^{-1}$ such that

(i) $\left(\gamma^{-1}\right)^{-1}=\gamma$ for all $\gamma \in \mathcal{G}$,

(ii) if $(\gamma, \xi),(\xi, \eta) \in \mathcal{G}^{(2)}$, then $(\gamma \xi, \eta),(\gamma, \xi \eta) \in \mathcal{G}^{(2)}$,

(iii) $\left(\gamma, \gamma^{-1}\right) \in \mathcal{G}^{(2)}$ for all $\gamma \in \mathcal{G}$,

(iv) for all $(\gamma, \xi) \in \mathcal{G}^{(2)},(\gamma \xi) \xi^{-1}=\gamma$ and $\gamma^{-1}(\gamma \xi)=\xi$.

Given a groupoid we denote by $\mathcal{G}^{(0)}=\left\{\gamma \gamma^{-1}: \gamma \in \mathcal{G}\right\}$ the space of units and define the source and range maps $r, s: \mathcal{G} \rightarrow \mathcal{G}^{(0)}$ by the equations

$$
r(\gamma)=\gamma \gamma^{-1}, \quad s(\gamma)=\gamma^{-1} \gamma
$$

for all $\gamma \in \mathcal{G}$. The source and range maps allow us to characterise

$$
\mathcal{G}^{(2)}=\{(\gamma, \xi) \in \mathcal{G} \times \mathcal{G}: s(\gamma)=r(\xi)\} .
$$

Throughout this work, we will assume that $\mathcal{G}$ is equipped with second countable, locally compact and Hausdorff topology such that the mulitplication, inversion, source and range maps are all continuous. A groupoid $\mathcal{G}$ is étale if the range map $r: \mathcal{G} \rightarrow \mathcal{G}^{(0)}$ is a local homeomorphism. Étale groupoids have the useful property that for all $x \in \mathcal{G}^{(0)}$, the fibres $r^{-1}(x)$ and $s^{-1}(x)$ are discrete.

Examples 2.16 (i) Let $G$ be a group, then it is also a groupoid such that $G^{(0)}=\{e\}$ with multiplication and inverse given by the group operation. If $G$ is discrete, then it is étale as a groupoid.

(ii) Let $X$ be a locally compact Hausdorff space, $G$ a locally compact group and suppose there is a continuous left-action $G \rightarrow \operatorname{Homeo}(X)$ so that $(g, x) \mapsto$ $g \cdot x$ is jointly continuous. We can define the locally compact and Hausdorff transformation groupoid $X \rtimes G$ given by pairs $(x, g) \in X \times G$ such that $(X \rtimes$ $G)^{(0)}=X$,

$$
\begin{aligned}
(x, g)^{-1} & =\left(g^{-1} \cdot x, g^{-1}\right), & & (x, g)\left(g^{-1} \cdot x, h\right)=(x, g h), \\
s(x, g) & =g^{-1} \cdot x, & & r(x, g)=x .
\end{aligned}
$$

Definition 2.17 Let $\mathcal{G}$ be a locally compact and Hausdorff groupoid. A continuous $\operatorname{map} \sigma: \mathcal{G}^{(2)} \rightarrow \mathbb{T}$ is a 2-cocycle if

$$
\sigma(\gamma, \xi) \sigma(\gamma \xi, \eta)=\sigma(\gamma, \xi \eta) \sigma(\xi, \eta)
$$

for any $(\gamma, \xi),(\xi, \eta) \in \mathcal{G}^{(2)}$, and

$$
\sigma(\gamma, s(\gamma))=1=\sigma(r(\gamma), \gamma)
$$


for all $\gamma \in \mathcal{G}$. We will call a groupoid 2-cocycle normalised if $\sigma\left(\gamma, \gamma^{-1}\right)=1$ for all $\gamma \in \mathcal{G}$.

The following result is well-known, though for completeness we provide a proof.

Lemma 2.18 If a groupoid 2-cocycle $\sigma: \mathcal{G}^{(2)} \rightarrow \mathbb{T}$ is normalised, then $\sigma(\gamma, \xi)=$ $\overline{\sigma\left(\gamma \xi, \xi^{-1}\right)}$ and $\sigma(\gamma, \xi)=\overline{\sigma\left(\xi^{-1}, \gamma^{-1}\right)}$ for all $(\gamma, \xi) \in \mathcal{G}^{(2)}$.

Proof Using the 2-cocyle identity,

$$
\sigma(\gamma, \xi) \sigma\left(\gamma \xi, \xi^{-1}\right)=\sigma\left(\gamma, s\left(\xi^{-1}\right)\right) \sigma\left(\xi, \xi^{-1}\right)=1
$$

so $\sigma(\gamma, \xi)=\overline{\sigma\left(\gamma \xi, \xi^{-1}\right)}$. Next we compute that

$$
\sigma\left(\gamma \xi, \xi^{-1}\right) \sigma\left(\gamma \xi \xi^{-1}, \gamma^{-1}\right)=\sigma\left(\gamma \xi, \xi^{-1} \gamma^{-1}\right) \sigma\left(\xi^{-1}, \gamma^{-1}\right)=\sigma\left(\xi^{-1}, \gamma^{-1}\right)
$$

and using the first identity

$$
\begin{aligned}
& \sigma\left(\gamma \xi, \xi^{-1}\right) \sigma\left(\gamma \xi \xi^{-1}, \gamma^{-1}\right) \\
& \quad=\overline{\sigma(\gamma, \xi) \sigma\left(\gamma \xi \xi^{-1} \gamma^{-1}, \gamma\right)}=\overline{\sigma(\gamma, \xi) \sigma(r(\gamma \xi), \gamma)}=\overline{\sigma(\gamma, \xi)}
\end{aligned}
$$

which gives that $\overline{\sigma(\gamma, \xi)}=\sigma\left(\xi^{-1}, \gamma^{-1}\right)$.

We briefly review the construction of groupoid $C^{*}$-algebras.

Definition 2.19 A Haar system on a locally compact Hausdorff groupoid $\mathcal{G}$ is a set of measures $\left\{v^{x}: x \in \mathcal{G}^{(0)}\right\}$ on $\mathcal{G}$ such that $\operatorname{supp}\left(v^{x}\right)=r^{-1}(x)$ and for all $f \in C_{c}(\mathcal{G})$,

$$
\int_{\mathcal{G}} f(\xi) \mathrm{d} \nu^{r(\eta)}(\xi)=\int_{\mathcal{G}} f(\eta \xi) \mathrm{d} \nu^{s(\eta)}(\xi), \quad g(x):=\int_{\mathcal{G}} f(\xi) \mathrm{d} \nu^{x}(\xi) \in C\left(\mathcal{G}^{(0)}\right) .
$$

Étale groupoids always have a Haar system given by the counting measure on the (discrete) fibres $r^{-1}(x)$. Given $\mathcal{G}$ with a 2-cocycle $\sigma$ and Haar system $\left\{v^{x}\right\}_{x \in \mathcal{G}^{(0)}}$, we can define a $*$-algebra structure on $C_{c}(\mathcal{G}, \sigma)$,

$\left(f_{1} * f_{2}\right)(\eta)=\int_{\mathcal{G}} f(\xi) g\left(\xi^{-1} \eta\right) \sigma\left(\xi, \xi^{-1} \eta\right) \mathrm{d} \nu^{r(\eta)}(\xi), \quad f^{*}(\xi)=\sigma\left(\xi, \xi^{-1}\right) \overline{f\left(\xi^{-1}\right)}$.

We will restrict ourselves to normalised cocycles, $\sigma\left(\xi, \xi^{-1}\right)=1$ as it covers all examples of interest to us. See [36] for the general construction. The algebra $C_{c}(\mathcal{G}, \sigma)$ has a right $C_{0}\left(\mathcal{G}^{(0)}\right)$-module structure, where $(f \cdot g)(\xi)=f(\xi) g(s(\xi))$ for $f \in$ $C_{c}(\mathcal{G}, \sigma)$ and $g \in C_{0}\left(\mathcal{G}^{(0)}\right)$. We can define a $C_{0}\left(\mathcal{G}^{(0)}\right)$-valued inner-product

$$
\begin{aligned}
\left(f_{1} \mid f_{2}\right)_{\mathcal{G}^{(0)}}(x) & =\int_{\mathcal{G}} \overline{f_{1}\left(\xi^{-1}\right)} f_{2}\left(\xi^{-1}\right) \sigma\left(\xi, \xi^{-1}\right) \mathrm{d} \nu^{x}(\xi) \\
& =\int_{\mathcal{G}} \overline{f_{1}\left(\xi^{-1}\right)} f_{2}\left(\xi^{-1}\right) \mathrm{d} \nu^{x}(\xi)
\end{aligned}
$$


as $\sigma$ is normalised. Completing this space in the norm of $C_{0}\left(\mathcal{G}^{(0)}\right)$ gives a right$C_{0}\left(\mathcal{G}^{(0)}\right)$-module, which we denote by $L^{2}(\mathcal{G}, v)_{\mathcal{G}^{(0)}}$. There is a canonical left-action of $C_{c}(\mathcal{G}, \sigma)$ on $L^{2}(\mathcal{G}, v)_{\mathcal{G}^{(0)}}$ by the (twisted) convolution product.

Definition 2.20 (cf. [22]) The reduced groupoid $C^{*}$-algebra $C_{r}^{*}(\mathcal{G}, \sigma)$ is the completion of $C_{c}(\mathcal{G}, \sigma)$ in the norm inherited from the embedding $C_{c}(\mathcal{G}, \sigma) \hookrightarrow$ End $_{C_{0}\left(\mathcal{G}^{(0)}\right)}^{*}\left(L^{2}(\mathcal{G}, v)\right)$.

If there is a topological space $Z$ with (continuous) map $\rho: Z \rightarrow \mathcal{G}^{(0)}$, we denote by $\mathcal{G} \ltimes_{\rho} Z$ and $Z \rtimes_{\rho} \mathcal{G}$ the pullback with respect to the source and range maps respectively.

Definition 2.21 ( $\mathcal{G}$-space) A Hausdorff topological space $Z$ is a left $\mathcal{G}$-space if there is a continuous map, called the anchor or moment map, $\rho: Z \rightarrow \mathcal{G}^{(0)}$ and a continuous map

$$
\mathcal{G} \ltimes_{\rho} Z \rightarrow Z, \quad(\gamma, z) \mapsto \gamma \cdot z \in Z
$$

such that for $(\gamma, z) \in \mathcal{G} \ltimes_{\rho} Z$ and $\left(\gamma_{1}, \gamma_{2}\right) \in \mathcal{G}^{(2)}$,

$$
\rho(\gamma \cdot z)=r(\gamma), \quad \rho(z) \cdot z=z, \quad\left(\gamma_{1} \gamma_{2}\right) \cdot z=\gamma_{1} \cdot\left(\gamma_{2} \cdot z\right)
$$

Unless otherwise stated, we will always assume that the moment map $\rho: Z \rightarrow \mathcal{G}^{(0)}$ is open and surjective. One may also consider a right $\mathcal{H}$-space from $\phi: Z \rightarrow \mathcal{H}^{(0)}$, where the definition is analogous to the above but instead using a map $Z \rtimes_{\phi} \mathcal{H} \rightarrow Z$ such that $\rho(z \cdot \eta)=s(\eta)$. When the context is clear, we will write left/right-actions as $\gamma z$ or $z \eta$.

We say that $Z$ is a proper $\mathcal{G}$-space (or that $\mathcal{G}$ acts properly) if the map

$$
\mathcal{G} \ltimes_{\rho} Z \rightarrow Z \times Z, \quad(\gamma, z) \mapsto(\gamma \cdot z, z)
$$

is proper. If the map $(\gamma, z) \mapsto(\gamma \cdot z, z)$ is injective, then we say that $Z$ is a free $\mathcal{G}$-space or that $\mathcal{G}$ acts freely.

Definition 2.22 Let $\mathcal{G}$ and $\mathcal{H}$ be (locally compact, Hausdorff) groupoids and assume that $Z$ carries both a left- $\mathcal{G}$ and right- $\mathcal{H}$ action via moment maps $\rho: Z \rightarrow \mathcal{G}^{(0)}$ and $\phi: Z \rightarrow \mathcal{H}^{(0)}$. We say that $Z$ is a $\mathcal{G}-\mathcal{H}$-bibundle if the actions commute, i.e.,

(1) for all $(\gamma, z) \in \mathcal{G} \ltimes_{\rho} Z$ and $(z, \eta) \in Z \rtimes_{\phi} \mathcal{H},(\gamma \cdot z) \cdot \eta=\gamma \cdot(z \cdot \eta)$,

(2) for all $(z, \eta) \in Z \rtimes_{\phi} \mathcal{H}, \rho(z \cdot \eta)=\rho(z)$,

(3) for all $(\gamma, z) \in \mathcal{G} \ltimes_{\rho} Z, \phi(\gamma \cdot z)=\phi(z)$.

A $\mathcal{G}-\mathcal{H}$ bibundle is a groupoid equivalence if the maps

$$
\begin{array}{ll}
\mathcal{G} \ltimes_{\rho} Z \rightarrow Z *_{\mathcal{H}^{(0)} Z,} & (\gamma, z) \mapsto(\gamma \cdot z, z), \\
Z \rtimes_{\phi} \mathcal{H} \rightarrow Z *_{\mathcal{G}^{(0)} Z,} & (z, \eta) \mapsto(z, z \cdot \eta)
\end{array}
$$

are homeomorphisms. 
At the level of operator algebras, groupoid equivalence implies Morita equivalence of the (full or reduced) groupoid $C^{*}$-algebras [34,38]. Let $\mathcal{G} \stackrel{\rho}{\leftarrow} Z \stackrel{\phi}{\rightarrow} \mathcal{H}$ be a groupoid equivalence such that $\mathcal{G}$ and $\mathcal{H}$ have Haar systems $\left\{v^{x}\right\}_{x \in \mathcal{G}^{(0)}}$ and $\left\{\lambda^{y}\right\}_{y \in \mathcal{H}^{(0)}}$ respectively. Then there is a left (resp. right) action of $C_{c}(\mathcal{G})\left(\right.$ resp. $\left.C_{c}(\mathcal{H})\right)$ on $C_{c}(Z)$ given by

$$
\begin{aligned}
& (f \cdot \xi)(z)=\int_{\mathcal{G}} f(\gamma) \xi\left(\gamma^{-1} z\right) \mathrm{d} \nu^{\rho(z)}(\gamma), \quad \xi \in C_{c}(Z), \quad f \in C_{c}(\mathcal{G}), \\
& (\xi \cdot g)(z)=\int_{\mathcal{H}} \xi(z \eta) g\left(\eta^{-1}\right) \mathrm{d} \lambda^{\phi(z)}(\eta), \quad \xi \in C_{c}(Z), \quad g \in C_{c}(\mathcal{H}) .
\end{aligned}
$$

There is also the $C_{c}(\mathcal{H})$-valued inner product

$$
\left(\xi_{1} \mid \xi_{2}\right) \mathcal{H}(\eta)=\int_{\mathcal{G}} \overline{\xi_{1}\left(\gamma^{-1} z\right)} \xi_{2}\left(\gamma^{-1} z \eta\right) \mathrm{d} \nu^{\rho(z)}(\gamma)
$$

where $z \in Z$ is chosen such that $\phi(z)=r(\eta)$.

Proposition 2.23 [38, Theorem 4.1] Let $Z$ be a $\mathcal{G}-\mathcal{H}$ groupoid equivalence. Then $C_{c}(Z)$ is a pre-Morita equivalence bimodule for $C_{c}(\mathcal{G})$ and $C_{c}(\mathcal{H})$. Consequently, $C_{r}^{*}(\mathcal{G})$ and $C_{r}^{*}(\mathcal{H})$ are Morita equivalent.

We denote by $\mathcal{G}^{2}(Z)_{\mathcal{H}}$ the Morita equivalence bimodule that links $C_{r}^{*}(\mathcal{G})$ and $C_{r}^{*}(\mathcal{H})$. Because we work with reduced groupoid $C^{*}$-algebras, the Morita equivalence bimodule completion of $C_{c}(Z)$ is constructed from the linking groupoid $L=\mathcal{G} \sqcup Z \sqcup$ $Z^{\mathrm{op}} \sqcup \mathcal{H}$ [38]. In Sect. 4.1 we discuss instances of twisted groupoid Morita equivalence.

\section{Frames of Translates and Wannier Bases from Groupoid Equivalences}

We let $\mathcal{G} \stackrel{\rho}{\leftarrow} Z \stackrel{\phi}{\rightarrow} \mathcal{H}$ be a $\mathcal{G}-\mathcal{H}$ equivalence of locally compact, second countable and Hausdorff groupoids such that $\mathcal{H}^{(0)}$ is compact and $\mathcal{H}$ is étale. In particular, this implies that $C_{r}^{*}(\mathcal{H})$ is unital. We assume that $\mathcal{G}$ has a Haar system $\left\{v^{x}\right\}_{x \in \mathcal{G}^{(0)}}$ and so $C_{c}\left(\mathcal{G )} C_{c}(Z)_{C_{c}(\mathcal{H})}\right.$ is a pre-Morita equivalence bimodule described by Eqs. (6) and (7). Because $\mathcal{H}$ is étale, the right-action in Eq. (6) reduces to a sum over the discrete set $r^{-1}(\phi(z))$.

\subsection{C*-Module Frame}

Because all $C^{*}$-algebras are separable, there exists a countable right module frame for $\mathcal{G}^{2}(Z)_{\mathcal{H}}$. Furthermore, since $C_{r}^{*}(\mathcal{H})$ is unital, $\mathcal{G} L^{2}(Z)_{\mathcal{H}}$ is finitely generated and projective as a left $C_{r}^{*}(\mathcal{G})$-module and so has a finite left $C^{*}$-module frame.

A right module frame for the submodule $C_{c}(Z)$ is constructed in [34, Proposition 2.10]. We briefly review this construction. We say that a subset $L \subset \mathcal{G}$ is $r$-relatively compact if $L \cap r^{-1}(K)$ is relatively compact for every compact $K \subset \mathcal{G}$. We consider 
a triple $(K, U, \varepsilon)$ with $K \subset \mathcal{G}^{(0)}$ compact with $U \subset \mathcal{G}$ an $r$-relatively compact neighbourhood of $\mathcal{G}^{(0)}$ and $\varepsilon>0$. Because $\mathcal{G}^{(0)}$ is paracompact and $\mathcal{G}$ acts properly on $Z$, there are open, relatively compact sets $\left\{V_{j}\right\}_{j=1}^{n} \subset Z$ such that $\left\{\rho\left(V_{j}\right)\right\}_{j=1}^{n}$ cover $K$ and are such that $(\gamma z, z) \in V_{j} \times V_{j}$ implies that $\gamma \in U$. We take a partition of unity $\left\{\psi_{j}\right\}_{j=1}^{n}$ subordinate to the cover $\left\{\rho\left(V_{j}\right)\right\}_{j=1}^{N}$. We can then find functions $\left\{\tilde{\psi}_{j}\right\}_{j=1}^{n}$ $\operatorname{such}$ that $\operatorname{supp}\left(\tilde{\psi}_{j}\right) \subset V_{j}$ and

$$
\sum_{\eta \in r^{-1}(\phi(z))} \tilde{\psi}_{j}(z \eta)=\psi_{j}(\rho(z))
$$

Finally, we can approximate $\tilde{\psi}_{j}$ with $\left\{\varphi_{j}\right\}_{j=1}^{n}$ such that $\operatorname{supp}\left(\varphi_{j}\right) \subset V_{j}$ and

$$
\left|\tilde{\psi}_{j}(z)-\varphi_{j}(z) \int_{\mathcal{G}} \varphi_{j}\left(\gamma^{-1} z\right) \mathrm{d} \nu^{\rho(z)}(\gamma)\right| \leq \frac{\varepsilon}{M} \quad M=\sup _{z} \sum_{j=1}^{n} \sum_{\eta \in r^{-1}(\phi(z))} \chi_{V_{j}}(z \eta) .
$$

The functions $e_{n}=\sum_{j=1}^{n} \mathcal{G}\left(\varphi_{j} \mid \varphi_{j}\right)$ are an approximate identity for the left action of $C_{c}(\mathcal{G})$ on $C_{c}(Z)$. The functions $\left\{\varphi_{j}\right\} \subset C_{c}(Z)$ can then be used to construct a right $C^{*}$-module frame in the Morita equivalence bimodule $\mathcal{G}^{2}(Z)_{\mathcal{H}}$ [38].

\subsection{Localisation and Hilbert Space Frame}

For any $x \in \mathcal{H}^{(0)}$ we have a state $\omega_{x}$ given by the restriction of $f \in C_{c}(\mathcal{H})$ to $\mathcal{H}^{(0)}$ and then evaluation at $x \in \mathcal{H}^{(0)}$. We see that

$$
\omega_{x}\left(g^{*} g\right)=\sum_{\eta \in r^{-1}(x)}|g(\eta)|^{2},
$$

which shows that $\omega_{x}$ is positive. There are other possible states on $C_{r}^{*}(\mathcal{H})$ that one may also consider such as integration with respect to a quasi-invariant measure [36]. The reason we choose the evaluation state is because we would like to construct Parseval frames that have a discrete labeling. By choosing $x \in \mathcal{H}^{(0)}$, the discrete set $r^{-1}(x)$ provides us with such a labeling.

Lemma 3.1 Fix an element $x \in \mathcal{H}^{(0)}$. Then there are real-valued functions $\left\{\delta_{\alpha}\right\}_{\alpha \in r^{-1}(x)} \subset C_{c}(\mathcal{H})$ such that $\omega_{x}\left(\delta_{\alpha_{1}} * \delta_{\alpha_{2}}^{*}\right)=\delta_{\alpha_{1}, \alpha_{2}}$.

Proof Because $\mathcal{H}^{(0)}$ is compact and $r^{-1}(x)$ is discrete, for each $\alpha \in r^{-1}(x)$ we take $\delta_{\alpha}$ a bump function supported on $U_{\alpha}$ such that $U_{\alpha} \cap r^{-1}(x)=\{\alpha\}$ and $\delta_{\alpha}(\alpha)=1$. Then we compute that

$$
\omega_{x}\left(\delta_{\alpha_{1}} * \delta_{\alpha_{2}}^{*}\right)=\sum_{\beta \in r^{-1}(x)} \delta_{\alpha_{1}}(\beta) \delta_{\alpha_{2}}(\beta)=\delta_{\alpha_{2}}\left(\alpha_{1}\right)=\delta_{\alpha_{1}, \alpha_{2}}
$$

as the sum vanishes everywhere except for at most one term. 
Lemma 3.2 Let $Z_{x}=\phi^{-1}(x)$ and for $z \in Z_{x}$ define the measure $v^{\rho(z)}$ on $Z_{x}$ by

$$
\int_{Z_{x}} f(w) \mathrm{d} \nu^{\rho(z)}(w):=\int_{\mathcal{G}} f\left(\gamma^{-1} \cdot z\right) \mathrm{d} \nu^{\rho(z)}(\gamma)
$$

The Hilbert space localisation $\mathfrak{h}_{x}$ of $\mathcal{G}^{2} L^{2}(Z)_{\mathcal{H}}$ in $\omega_{x}$ is $L^{2}\left(Z_{x}, \mathrm{~d} \nu^{\rho(z)}\right)$ with $z \in Z_{x}$ chosen arbitrarily.

Proof We consider the inner-product, where

$$
\begin{aligned}
\left\langle e_{1}, e_{2}\right\rangle_{x} & =\omega_{x}\left(\left(e_{1} \mid e_{2}\right) \mathcal{H}\right)=\left(e_{1} \mid e_{2}\right) \mathcal{H}(x)=\int_{\mathcal{G}} \overline{e_{1}\left(\gamma^{-1} z\right)} e_{2}\left(\gamma^{-1} z x\right) \mathrm{d} \nu^{\rho(z)}(\gamma) \\
& =\int_{\mathcal{G}} \overline{e_{1}\left(\gamma^{-1} z\right)} e_{2}\left(\gamma^{-1} z \phi(z)\right) \mathrm{d} \nu^{\rho(z)}(\gamma)=\int_{\mathcal{G}} \overline{e_{1}\left(\gamma^{-1} z\right)} e_{2}\left(\gamma^{-1} z\right) \mathrm{d} \nu^{\rho(z)}(\gamma)
\end{aligned}
$$

Hence the Hilbert space is the $L^{2}$-completion of $C_{c}\left(Z_{x}\right)$ with respect to the measure $v^{\rho(z)}$. Since $Z$ is an equivalence, for every $z, w \in Z_{x}$ there exists $\gamma \in r^{-1}(\rho(z)) \subset \mathcal{G}$ such that $w=\gamma^{-1} z$. The measure $\nu^{\rho(z)}$ is thus independent of the choice of $z \in Z_{x}$.

Given $x \in \mathcal{H}^{(0)}$ and $e \in \mathcal{G}^{2}(Z)_{\mathcal{H}}$, we let $e_{x}$ be the corresponding element in the localisation $L^{2}\left(Z_{x}\right)$. Given any $\alpha \in r^{-1}(x)$, define a function $e_{x}^{\alpha} \in L^{2}\left(Z_{x}\right)$ by $\left(e_{x}^{\alpha}\right)(y)=e(y \alpha), y \in Z_{x}$.

Lemma 3.3 Let $e \in \mathcal{G}^{2}(Z)_{\mathcal{H}}, a \in \mathcal{H}^{(0)}$ and $\alpha \in r^{-1}(x)$.

(i) There is an equality $e_{x}^{\alpha}=\left(e \cdot \delta_{\alpha}^{*}\right)_{x}$ with $\delta_{\alpha}$ be the bump functions from Lemma 3.1.

(ii) If $e \in \mathcal{G} L^{2}(Z)_{\mathcal{H}}$ is such that $(e \mid e)_{\mathcal{H}}=1_{\mathcal{H}}$, then $\left\{e_{x}^{\alpha}\right\}_{\alpha \in r^{-1}(x)}$ is an orthonormal system in $L^{2}\left(Z_{x}\right)$;

(iii) Given $e_{1}, e_{2}, \xi \in \mathcal{G}^{2}(Z)_{\mathcal{H}}$,

$$
\left(\Theta_{e_{1}, e_{2}}^{R}(\xi)\right)_{x}=\sum_{\alpha \in r^{-1}(x)}\left(e_{1}\right)_{x}^{\alpha}\left\langle\left(e_{2}\right)_{x}^{\alpha}, \xi_{x}\right\rangle_{x} .
$$

Proof. We first note that $e(y \alpha)$ is well-defined as $\rho(y)=x=r(\alpha)$. For part (i) we compute for $y \in Z_{x}$,

$$
\left(e \cdot \delta_{\alpha}^{*}\right)(y)=\sum_{\beta \in r^{-1}(x)} e(y \beta) \delta_{\alpha}^{*}\left(\beta^{-1}\right)=\sum_{\beta \in r^{-1}(x)} e(y \beta) \delta_{\alpha}(\beta)=e(y \alpha) .
$$

Using part (i) and Lemma 3.1, we see that for $e$ such that $(e \mid e)_{\mathcal{H}}=1$,

$$
\left\langle e_{x}^{\alpha_{1}}, e_{x}^{\alpha_{2}}\right\rangle_{x}=\omega_{x}\left(\left(e \cdot \delta_{\alpha_{1}}^{*} \mid e \cdot \delta_{\alpha_{2}}^{*}\right)_{\mathcal{H}}\right)=\omega_{x}\left(\delta_{\alpha_{1}}(e \mid e)_{\mathcal{H}} \delta_{\alpha_{2}}^{*}\right)=\delta_{\alpha_{1}, \alpha_{2}}
$$

and so $\left\{e_{x}^{\alpha}\right\}_{\alpha \in r^{-1}(x)}$ is an orthonormal system, which proves part (ii). 
For part (iii), we again compute for $y \in \phi^{-1}(x)$

$$
\begin{aligned}
\left(\Theta_{e_{1}, e_{2}}^{R}(\xi)\right)(y) & =\sum_{\alpha \in r^{-1}(x)} e_{1}(y \alpha)\left(e_{2} \mid \xi\right) \mathcal{H}\left(\alpha^{-1}\right) \\
& =\sum_{\alpha \in r^{-1}(x)} e_{1}(y \alpha) \int_{\mathcal{G}} \overline{e_{2}\left(\gamma^{-1} z\right)} \xi\left(\gamma^{-1} z \alpha^{-1}\right) \mathrm{d} \nu^{\rho(z)}(\gamma) .
\end{aligned}
$$

We now let $u=z \alpha^{-1}$, where $u \alpha=z s(\alpha)=z \phi(z)=z$ and $\rho(u)=\rho\left(z \alpha^{-1}\right)=\rho(z)$ as $Z$ is a groupoid equivalence. Hence

$$
\begin{aligned}
\left(\Theta_{e_{1}, e_{2}}^{R}(\xi)\right)(y) & =\sum_{\alpha \in r^{-1}(x)} e_{1}(y \alpha) \int_{\mathcal{G}} \overline{e_{2}\left(\gamma^{-1} u \alpha\right)} \xi\left(\gamma^{-1} u\right) \mathrm{d} \nu^{\rho(u)}(\gamma) \\
& =\sum_{\alpha \in r^{-1}(x)}\left(e_{1}\right)_{x}^{\alpha}(y)\left\langle\left(e_{2}\right)_{x}^{\alpha}, \xi_{x}\right\rangle_{x} .
\end{aligned}
$$

This completes the proof.

For a countable set $J$ and a $C^{*}$-algebra $B$ we denote by $\ell^{2}(J, B)$ the standard Hilbert $C^{*}$-module of sequences indexed by $J$. That is

$$
\ell^{2}(J, B):=\left\{f: J \rightarrow B: \sum_{j \in J} f(j)^{*} f(j)<\infty\right\},
$$

where the series converges in $B$. We now come to our main result relating $C^{*}$-module frames to localised frames of translates.

Theorem 3.4 Let $\left\{e_{j}\right\}_{j \in J} \subset \mathcal{G}^{2}(Z)_{\mathcal{H}}$ be a countable subset and $E:=$

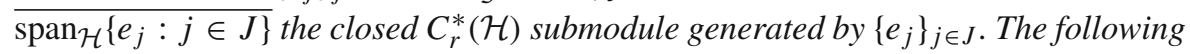
are equivalent:

(1) The sequence $\left\{e_{j}\right\}_{j \in J}$ is a right $C^{*}$-module frame of $E \subset \mathcal{G}^{2}(Z)_{\mathcal{H}}$;

(2) For $\xi \in E$ the map $j \mapsto\left(e_{j} \mid \xi\right)_{\mathcal{H}}$ takes values in $\ell^{2}\left(J, C_{r}^{*}(\mathcal{H})\right)$ and for all $x \in \mathcal{H}^{(0)}$ the set $\left\{\left(e_{j}\right)_{x}^{\alpha}\right\}_{j \in J, \alpha \in r^{-1}(x)}$ is a normalised tight frame for $\overline{E_{x}} \subset L^{2}\left(Z_{x}\right)$;

Proof $(1) \Rightarrow(2)$ : Using part (iii) of Lemma 3.3, we see that

$$
\sum_{j \in J} \sum_{\alpha \in r^{-1}(x)}\left(e_{j}\right)_{x}^{\alpha}\left\langle\left(e_{j}\right)_{x}^{\alpha}, \xi_{x}\right\rangle_{x}=\sum_{j \in J}\left(\Theta_{e_{j}, e_{j}}^{R}(\xi)\right)_{x}=\xi_{x}
$$

as $\left(e_{j}\right)_{j \in J}$ is a right $C^{*}$-module frame.

$(2) \Rightarrow(1)$ : In order to prove that $\left\{e_{j}\right\}_{j \in J}$ is a Hilbert $C^{*}$-module frame for $E$, we need to show that the map

$$
v: E \rightarrow \ell^{2}\left(J, C_{r}^{*}(\mathcal{H})\right), \quad \xi \mapsto\left(e_{j} \mid \xi\right)_{\mathcal{H}},
$$


satisfies $v^{*} v=1$. Note that $v$ is well-defined since we assume that $j \mapsto\left(e_{j} \mid \xi\right)_{\mathcal{H}}$ is an element of $\ell^{2}\left(J, C_{r}^{*}(\mathcal{H})\right)$, and $v$ is automatically adjointable with $v^{*}\left(b_{j}\right)=\sum_{j} e_{j} \cdot b_{j}$. Since $\left\{\left(e_{j}\right)_{x}^{\alpha}\right\}_{j \in J, \alpha \in r^{-1}(x)}$ is a normalised tight frame for $\overline{E_{x}} \subset L^{2}\left(Z_{x}\right)$, the map

$$
v_{x}: L^{2}\left(Z_{x}\right) \rightarrow \ell^{2}\left(J \times r^{-1}(x)\right), \quad \psi \mapsto\left\langle\left(e_{j}\right)_{x}^{\alpha}, \psi\right\rangle_{x},
$$

is an isometry, for

$$
\begin{aligned}
\langle\psi, \psi\rangle_{x} & =\|\psi\|_{x}^{2}=\sum_{(j, \alpha) \in J \times r^{-1}(x)}\left|\left\langle\left(e_{j}\right)_{x}^{\alpha}, \psi\right\rangle_{x}\right|^{2}=\left\|v_{x}(\psi)\right\|^{2} \\
& =\left\langle v_{x} \psi, v_{x} \psi\right\rangle_{\ell^{2}\left(J \times r^{-1}(x)\right)},
\end{aligned}
$$

and it follows that $v_{x}^{*} v_{x}=1$. The map $v_{x}^{*}$ is given by

$$
v_{x}^{*}:\left(\lambda_{j}^{\alpha}\right) \mapsto \sum_{j, \alpha}\left(e_{j}\right)_{x}^{\alpha} \lambda_{j}^{\alpha}
$$

and we find from Lemma 3.3

$$
\xi_{x}=v_{x}^{*} v_{x}(\psi)=\sum_{\alpha, j}\left(e_{j}\right)_{x}^{\alpha}\left\langle\left(e_{j}\right)_{x}^{\alpha}, \xi_{x}\right\rangle_{x}=\left(\sum_{j} \Theta_{e_{j}, e_{j}}(\xi)\right)_{x}=\left(v^{*} v(\xi)\right)_{x} .
$$

Therefore we can conclude that

$$
\left\|\xi-v^{*} v(\xi)\right\|=\sup _{x}\left\|\xi-v^{*} v(\xi)\right\|_{x}=\sup _{x}\left\|\xi_{x}-\left(v^{*} v(\xi)\right)_{x}\right\|_{x}=0
$$

and hence $v^{*} v=1$.

Remark 3.5 Well-definedness of the map $v: E \rightarrow \ell^{2}\left(J, C_{r}^{*}(\mathcal{H})\right)$ entails that for all $e \in \mathcal{E}$ the series

$$
\sum_{j}\left(e \mid e_{j}\right)_{\mathcal{H}}\left(e_{j} \mid e\right)_{\mathcal{H}}
$$

is norm convergent in $C_{r}^{*}(\mathcal{H})$. This is automatic when the index set $J$ is finite but poses a non-trivial restriction for infinite $J$.

\subsection{Finitely Generated and Projective Modules}

Using the left-action of $C_{r}^{*}(\mathcal{G})$ on $L^{2}(Z)$, we can define a representation of $\pi_{x}$ : $C_{r}^{*}(\mathcal{G}) \rightarrow \mathcal{B}\left(\mathfrak{h}_{x}\right)$, where

$$
\pi_{x}(f) \xi_{x}=(f \cdot \xi)_{x}, \quad f \in C_{r}^{*}(\mathcal{G}), \quad \xi \in L^{2}(Z) .
$$


Concretely,

$$
\left(\pi_{x}(f) \xi_{x}\right)(y)=\int_{\mathcal{G}} f(\gamma) \xi\left(\gamma^{-1} y\right) \mathrm{d} \nu^{\rho \circ \phi^{-1}(x)}(\gamma), \quad f \in C_{c}(\mathcal{G}), \quad \xi \in C_{c}(Z) .
$$

We consider projections, $p=p^{*}=p^{2} \in M_{n}\left(C_{r}^{*}(\mathcal{G})\right)$, which act compactly on $L^{2}(Z)^{\oplus n}$.

Proposition 3.6 Let $p=p^{*}=p^{2} \in M_{n}\left(C_{r}^{*}(\mathcal{G})\right)$. There is a finite set $\left\{v_{j}\right\}_{j=1}^{n} \subset$ $p L^{2}(Z)^{\oplus n}$ such that for any $x \in \mathcal{H}^{(0)},\left\{v_{1}^{\alpha}, \ldots, v_{n}^{\alpha}\right\}_{\alpha \in r^{-1}(x)}$ is a normalised tight frame of $\pi_{x}(p) \mathfrak{h}_{x}^{\oplus n}$.

Proof By Lemma 2.9 (2), there is a finite frame $\left\{v_{j}\right\}_{j=1}^{n}$ of $p L^{2}(Z)^{\oplus n}$. It is immediate that the localisation of $p L^{2}(Z)^{\oplus n}$ in $\omega_{x}$ is $\pi_{x}(p) \mathfrak{h}_{x}^{\oplus n}$. The result then follows by the same proof as Theorem 3.4.

Let us now consider the converse, i.e. given the Hilbert space frames $\left\{w_{1}^{\alpha}, \ldots\right.$, $\left.w_{m}^{\alpha}\right\}_{\alpha \in r^{-1}(x)}$ for $x \in \mathcal{H}^{(0)}$, we construct a finitely generated and projective module.

Proposition 3.7 Let $W \subset \mathcal{G}^{2}(Z)_{\mathcal{H}}$ be a closed submodule and $\left\{w_{1}, \ldots, w_{n}\right\}$ a finite subset of $\mathcal{G}^{2}(Z)_{\mathcal{H}}$ such that $W:=\overline{\operatorname{span}_{C_{r}^{*}(\mathcal{H})}\left\{w_{1}, \ldots, w_{n}\right\}}$. Suppose that for all $x \in \mathcal{H}^{(0)},\left\{w_{1}^{\alpha}, \ldots, w_{m}^{\alpha}\right\}_{\alpha \in r^{-1}(x)}$ is a normalised tight frame of $\overline{W_{x}} \subset L^{2}\left(Z_{x}, \mathrm{~d} v^{\rho(x)}\right)$. Then $W$ is a finitely generated and projective module over $C_{r}^{*}(\mathcal{H})$. If, for each $x \in \mathcal{H}^{(0)}$, $\left\{w_{1}^{\alpha}, \ldots, w_{m}^{\alpha}\right\}_{\alpha \in r^{-1}(x)}$ is an orthonormal basis, then $W \cong C_{r}^{*}(\mathcal{H})^{\oplus m}$.

Proof The first part of the Proposition follows immediately from Theorem 3.4 and the fact that any $C_{r}^{*}(\mathcal{H})$-module with a finite $C^{*}$-module frame is finitely generated and projective. Now suppose that $\left\{w_{1}^{\alpha}, \ldots, w_{m}^{\alpha}\right\}_{\alpha \in r^{-1}(x)}$ is an orthonormal basis and let $p_{j k}=\left(w_{j} \mid w_{k}\right)_{\mathcal{H}} \in C_{r}^{*}(\mathcal{H})$. For any $x \in \mathcal{H}^{(0)}$, we have that

$$
\begin{aligned}
\delta_{j, k} \delta_{\alpha_{1}, \alpha_{2}} & =\left\langle w_{j}^{\alpha_{1}}, w_{k}^{\alpha_{2}}\right\rangle_{x}=\omega_{x}\left(\delta_{\alpha_{1}}\left(w_{j} \mid w_{k}\right) \mathcal{H}_{\alpha_{2}}^{*}\right)=\sum_{\beta \in r^{-1}(x)}\left(\delta_{\alpha_{1}} * p_{j k}\right)(\beta) \delta_{\alpha_{2}}(\beta) \\
& =\left(\delta_{\alpha_{1}} * p_{j k}\right)\left(\alpha_{2}\right)=\sum_{\eta \in r^{-1}(x)} \delta_{\alpha_{1}}(\eta) p_{j k}\left(\eta^{-1} \alpha_{2}\right)=p_{j k}\left(\alpha_{1}^{-1} \alpha_{2}\right)
\end{aligned}
$$

for all $\alpha_{1}, \alpha_{2} \in r^{-1}(x)$ and all $j, k \in\{1, \ldots, m\}$. Now, for any $\eta \in \mathcal{H}$, we can find some $x \in \mathcal{H}^{(0)}$ and $\alpha, \beta \in r^{-1}(x)$ such that $\eta=\alpha^{-1} \beta$. Hence, $p_{j k}(\eta)=$ $p_{j k}\left(\alpha^{-1} \beta\right)=\delta_{j, k} \delta_{\alpha, \beta}$. This implies that the matrix $p \in M_{m}\left(C_{r}^{*}(\mathcal{H})\right)$ is the identity matrix. Hence $W \stackrel{\simeq}{\rightarrow} C_{r}^{*}(\mathcal{H})^{\oplus m}$.

Theorem 3.8 Let $p=p^{*}=p^{2} \in M_{n}\left(C_{r}^{*}(\mathcal{G})\right)$. The finitely generated and projective module $p L^{2}(Z)^{\oplus n}$ with frame $\left\{v_{j}\right\}_{j=1}^{m}$ is isomorphic to the free module $C_{r}^{*}(\mathcal{H})^{\oplus m}$ if and only iffor all $x \in \mathcal{H}^{(0)},\left\{v_{1}^{\alpha}, \ldots, v_{m}^{\alpha}\right\}_{\alpha \in r^{-1}(x)}$ is an orthonormal basis of $\pi_{x}(p) \mathfrak{h}_{x}^{\oplus n}$. 
Proof Suppose there is a unitary isomorphism of $C^{*}$-modules $\varphi: p L^{2}(Z)^{\oplus n} \stackrel{\widetilde{ }}{\rightarrow}$ $C_{r}^{*}(\mathcal{H})^{\oplus m}$. It is clear that $\left\{1_{j}\right\}_{j=1}^{m}$ is right-frame of $C_{r}^{*}(\mathcal{H})^{\oplus m}$ and because $\varphi$ respects the inner-product structure $\left\{v_{j}\right\}_{j=1}^{m}$ is a frame of $p L^{2}(Z)^{\oplus n}$ with $v_{j}=\varphi^{-1}\left(1_{j}\right)$. We know that $\left\{v_{j}^{\alpha}\right\}$ is a normalised tight frame by Proposition 3.6 and we see that

$$
\begin{aligned}
\left\langle\left(v_{j}\right)_{x}^{\alpha_{1}},\left(v_{k}\right)_{x}^{\alpha_{2}}\right\rangle_{x} & =\omega_{x}\left(\left(v_{j} \cdot \delta_{\alpha_{1}}^{*} \mid v_{k} \cdot \delta_{\alpha_{2}}^{*}\right) \mathcal{H}\right)=\omega_{x}\left(\delta_{\alpha_{1}}\left(\varphi^{-1}\left(1_{k}\right) \mid \varphi^{-1}\left(1_{j}\right)\right)_{\mathcal{H}} \delta_{\alpha_{2}}^{*}\right) \\
& =\delta_{j, k} \omega_{x}\left(\delta_{\alpha_{1}} * \delta_{\alpha_{2}}^{*}\right)=\delta_{j, k} \delta_{\alpha_{1}, \alpha_{2}} .
\end{aligned}
$$

Hence the tight frame is orthonormal and so is an orthonormal basis. The converse statement follows from Proposition 3.7.

Remark 3.9 ( $K$-theoretic interpretation) Theorem 3.8 has an interpretation via the $K$ theory of the groupoid $C^{*}$-algebras. Using the $*$-isomorphism $C_{r}^{*}(\mathcal{G}) \cong \mathbb{K}_{C_{r}^{*}(\mathcal{H})}\left(L^{2}(Z)\right)$, we can naturally consider any projection $p \in M_{n}\left(C_{r}^{*}(\mathcal{G})\right)$ as a finite-rank operator on $L^{2}(Z)_{\mathcal{H}}^{\oplus n}$. Therefore we can also consider $p$ as a projection in $M_{m}\left(C_{r}^{*}(\mathcal{H})\right)$ for some $m$, with corresponding $K$-theory class $[p] \in K_{0}\left(C_{r}^{*}(\mathcal{H})\right)$. If $p L^{2}(Z)_{\mathcal{H}} \cong C_{r}^{*}(\mathcal{H})^{\oplus m}$, then $[p]=m[1] \in K_{0}\left(C_{r}^{*}(\mathcal{H})\right)$ and the projection $p$ is trivial in reduced $K$-theory.

Let us now consider the case where there are dense pre- $C^{*}$-algebras $\mathcal{A} \subset C_{r}^{*}(\mathcal{G})$ and $\mathcal{B} \subset C_{r}^{*}(\mathcal{H})$, which will allow us to consider $C^{*}$-modules and Hilbert space frames with additional regularity as in Proposition 2.13. Thus suppose there are families of commuting $*$-derivations

$$
\left\{\partial_{j}^{\mathcal{H}}\right\}_{j=1}^{d}: C_{c}(\mathcal{H}) \rightarrow C_{c}(\mathcal{H}), \quad\left\{\partial_{j}^{\mathcal{G}}\right\}_{j=1}^{d}: C_{c}(\mathcal{G}) \rightarrow C_{c}(\mathcal{G}),
$$

as well as a family of maps $\nabla_{j}: C_{c}(Z) \rightarrow C_{c}(Z)$ such that for each $j$ and all $a \in C_{c}(\mathcal{G}), b \in C_{c}(\mathcal{H}), \xi, \eta \in C_{c}(Z)$, Eqs. (3) and (4) hold. Denote by $\mathcal{S}_{k}(\mathcal{G})$ and $\mathcal{S}_{k}(\mathcal{H})$ the degree $k$ Fréchet completions of $C_{c}(\mathcal{G})$ and $C_{c}(\mathcal{H})$ in these seminorms (Proposition 2.10), and by $\mathcal{S}_{k}(Z)$ the degree $k$ Fréchet completion of $C_{c}(Z)$. We often write $\mathcal{S}$ for $\mathcal{S}_{\infty}$ in each of these cases. By Proposition 2.13, $\mathcal{S}_{k}(\mathcal{G}) \mathcal{S}_{k}(Z) \mathcal{S}_{k}(\mathcal{H})$ is a pre-Morita equivalence bimodule for all $k=0, \ldots, \infty$.

Theorem 3.10 Let $k=0, \ldots, \infty$ and $p=p^{*}=p^{2} \in M_{n}\left(\mathcal{S}_{k}(\mathcal{G})\right)$. Then there is a finite frame $\left\{v_{j}\right\}_{j=1}^{m}$ of $p \mathcal{S}_{k}(Z)_{\mathcal{S}_{k}(\mathcal{H})}^{\oplus n}$ such that for all $x \in \mathcal{H}^{(0)}$ the normalised tight frame $\left\{v_{1}^{\beta}, \ldots, v_{m}^{\beta}\right\}_{\beta \in r^{-1}(x)}$ of $\pi_{x}(p) \mathfrak{h}_{x}^{\oplus n}$ is finite with respect to the seminorms $\|\cdot\|_{l, x}$ on $\mathfrak{h}_{x}$ for $0 \leq l \leq k$. There is an isomorphism $p \mathcal{S}_{k}(Z)_{\mathcal{S}_{k}(\mathcal{H})}^{\oplus n} \cong \mathcal{S}_{k}(\mathcal{H})^{\oplus m}$ if and only if for all $x \in \mathcal{H}^{(0)},\left\{v_{1}^{\beta}, \ldots, v_{m}^{\beta}\right\}_{\beta \in r^{-1}(x)}$ is an orthonormal basis.

Proof Because $v_{j} \cdot \delta_{\beta}^{*} \subset \mathcal{S}_{k}(Z)$ for any $x \in \mathcal{H}^{(0)}$ and $\beta \in r^{-1}(x)$, the first statement then follows from Lemma 2.9 and Proposition 2.13. Lemma 2.9 and the fact that $\mathcal{S}_{k}(\mathcal{H})$ is a pre- $C^{*}$-algebra give that $p \mathcal{S}_{k}(\mathcal{H})^{\oplus n}$ is a free $\mathcal{S}_{k}(\mathcal{H})$-module if and only if its $C^{*}$-completion is a free $C_{r}^{*}(\mathcal{H})$-module.

If $p \mathcal{S}_{k}(Z)_{\mathcal{S}_{k}(\mathcal{H})}^{\oplus n} \cong \mathcal{S}_{k}(\mathcal{H})^{\oplus m}$, then there is a finite frame $\left\{v_{1}, \ldots, v_{m}\right\} \subset p \mathcal{S}_{k}(Z)^{\oplus n}$

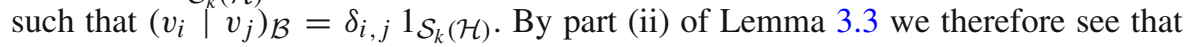
$\left\{v_{1}, \ldots, v_{m}^{\alpha}\right\}_{\alpha \in r^{-1}(x)}$ is an orthonormal system and hence must be an orthonormal 
basis. For the converse, we can use Proposition 3.7 and the fact that the finite $C_{r}^{*}(\mathcal{H})$ module frame can be approximated arbitrarily well by a finite $\mathcal{S}_{k}(\mathcal{H})$-module frame of the same size.

\section{Frames of Translates and Wannier Bases from Twisted Transversals}

Here we consider our framework in the case of groupoid equivalences that come from abstract transversals with an additional twist by a normalised groupoid 2-cocycle. In Sect. 5 we apply these results to the Delone transversal groupoid with twist coming from a magnetic field.

\subsection{Twisted Morita Equivalence}

Definition 4.1 A topological groupoid $\mathcal{G}$ admits an abstract transversal if there is a closed subset $X \subset \mathcal{G}^{(0)}$ such that

(i) $X$ meets every orbit of the $\mathcal{G}$-action on $\mathcal{G}^{(0)}$;

(ii) for the relative topologies on $X$ and

$$
\mathcal{G}_{X}:=\{\gamma \in \mathcal{G}: s(\gamma) \in X\} \subset \mathcal{G}
$$

the restrictions $r: \mathcal{G}_{X} \rightarrow \mathcal{G}^{(0)}$ and $s: \mathcal{G}_{X} \rightarrow X$ are open maps.

Given an abstract transversal $X \subset \mathcal{G}^{(0)}, \mathcal{G} \stackrel{r}{\leftarrow} \mathcal{G}_{X} \stackrel{s}{\rightarrow} \mathcal{H}$ is a $\mathcal{G}-\mathcal{H}$ groupoid equivalence for $\mathcal{H}=\left\{\gamma \in \mathcal{G}_{X}: r(\gamma) \in X\right\}$, see [34, Example 2.7]. Examples of abstract transversals include transitive groupoids and groupoids from foliations.

We now fix a locally compact, second countable and Hausdorff groupoid $\mathcal{G}$ such that $X \subset \mathcal{G}^{(0)}$ is compact and admits an abstract transversal $\mathcal{G}_{X}$ with $\mathcal{H}$ étale. We also fix a normalised groupoid 2-cocycle $\sigma_{\mathcal{G}}$ on $\mathcal{G}$, i.e. $\sigma_{\mathcal{G}}\left(\gamma, \gamma^{-1}\right)=1$ for all $\gamma \in \mathcal{G}$. The restriction of $\sigma_{\mathcal{G}}$ then gives a groupoid 2-cocycle $\sigma_{\mathcal{H}}$ for $\mathcal{H}$. The 2-cocycle twists the module structure

$$
\begin{aligned}
(f \cdot e)(z) & =\int_{\mathcal{G}} f(\gamma) e\left(\gamma^{-1} z\right) \sigma_{\mathcal{G}}\left(\gamma, \gamma^{-1} z\right) \mathrm{d} \nu^{r(z)}(\gamma), \quad e \in C_{c}\left(\mathcal{G}_{X}\right), f \in C_{c}\left(\mathcal{G}, \sigma_{\mathcal{G}}\right), \\
(e \cdot g)(z) & =\sum_{\eta \in r^{-1}(s(z))} e(z \eta) g\left(\eta^{-1}\right) \sigma_{\mathcal{G}}\left(z \eta, \eta^{-1}\right), \quad e \in C_{c}\left(\mathcal{G}_{X}\right), g \in C_{c}\left(\mathcal{H}, \sigma_{\mathcal{H}}\right), \\
\left(e_{1} \mid e_{2}\right)_{\mathcal{H}}(\eta) & =\int_{\mathcal{G}} \overline{e_{1}\left(\gamma^{-1} z\right)} e_{2}\left(\gamma^{-1} z \eta\right) \sigma_{\mathcal{G}}\left(z^{-1} \gamma, \gamma^{-1} z \eta\right) \mathrm{d} \nu^{r(z)}(\gamma), \quad r(\eta)=s(z) .
\end{aligned}
$$

Proposition 2.23 can be extended to the case of such simple twists. The case of general groupoid twists arising from $S^{1}$-extensions is handled via equivalence of Fell bundles, see $[33,39]$.

Proposition 4.2 The module $C_{c}\left(\mathcal{G}_{X}\right)$ is a pre-Morita equivalence bimodule for $C_{c}\left(\mathcal{G}, \sigma_{\mathcal{G}}\right)$ and $C_{c}\left(\mathcal{H}, \sigma_{\mathcal{H}}\right)$. Consequently, $C_{r}^{*}\left(\mathcal{G}, \sigma_{\mathcal{G}}\right)$ and $C_{r}^{*}\left(\mathcal{H}, \sigma_{\mathcal{H}}\right)$ are Morita equivalent. 


\subsection{Twisted Frames and Wannier Bases}

We now outline the minor changes required to recover the results of Sect. 3 to the case of twisted algebras. One advantage of restricting to normalised cocycles is that in the Hilbert space localisation, the inner-product simplifies. Namely, for $x \in X=\mathcal{H}^{(0)}$,

$$
\begin{aligned}
\left\langle\left(e_{1}\right)_{x},\left(e_{2}\right)_{x}\right\rangle_{x}=\left(e_{2} \mid e_{1}\right)_{\mathcal{H}}(x) & =\int_{\mathcal{G}} \overline{e_{2}\left(\gamma^{-1} z\right)} e_{1}\left(\gamma^{-1} z\right) \sigma_{\mathcal{G}}\left(z^{-1} \gamma, \gamma^{-1} z\right) \mathrm{d} \nu^{r o S^{-1}(x)}(\gamma) \\
& =\int_{\mathcal{G}} \overline{e_{2}\left(\gamma^{-1} z\right)} e_{1}\left(\gamma^{-1} z\right) \mathrm{d} \nu^{r \operatorname{Oos}^{-1}(x)}(\gamma) .
\end{aligned}
$$

Lemma 4.3 Let $x \in X$ and $\alpha \in r^{-1}(x)$. For $e_{x} \in \mathfrak{h}_{x}$, define $e_{x}^{\alpha}(y)=$ $e(y \alpha) \sigma_{\mathcal{G}}\left(y \alpha, \alpha^{-1}\right)$.

(i) If $e \in L^{2}\left(\mathcal{G}_{X}, \sigma\right)$ is such that $(e \mid e)_{\mathcal{H}}=1_{\mathcal{H}}$, then $\left\{e_{x}^{\alpha}\right\}_{\alpha \in r^{-1}(x)}$ is an orthonormal system in $\mathfrak{h}_{x}$.

(ii) Given $e_{1}, e_{2}, \xi \in \mathcal{G}^{2}\left(\mathcal{G}_{X}, \sigma\right)_{\mathcal{H}}$,

$$
\left(\Theta_{e_{1}, e_{2}}^{R}(\xi)\right)_{x}=\sum_{\alpha \in r^{-1}(x)}\left(e_{1}\right)_{x}^{\alpha}\left\langle\xi_{x},\left(e_{2}\right)_{x}^{\alpha}\right\rangle_{x}
$$

Proof. Like in the untwisted case, we see that for $y \in s^{-1}(x)$,

$$
\left(e \cdot \delta_{\alpha}^{*}\right)(y)=\sum_{\beta \in r^{-1}(x)} e(y \beta) \delta_{\alpha}(\beta) \sigma_{\mathcal{G}}\left(y \beta, \beta^{-1}\right)=e(y \alpha) \sigma_{\mathcal{G}}\left(y \alpha, \alpha^{-1}\right) .
$$

Hence we can compute

$$
\left\langle e_{x}^{\alpha_{1}}, e_{x}^{\alpha_{2}}\right\rangle_{x}=\omega_{x}\left(\delta_{\alpha_{1}} * \delta_{\alpha_{2}}^{*}\right)=\sum_{\beta \in r^{-1}(x)} \delta_{\alpha_{1}}(\beta) \delta_{\alpha_{2}}(\beta) \sigma_{\mathcal{H}}\left(\beta, \beta^{-1}\right)=\delta_{\alpha_{1}, \alpha_{2}} .
$$

For part (ii), can follow the same argument as Lemma 3.3. For $y \in s^{-1}(x)$ and $u=z \alpha^{-1}$ with $u \alpha=z s(\alpha)=z s(z)=z$ and $r(u)=r\left(z \alpha^{-1}\right)=r(z)$,

$$
\begin{aligned}
& \left(\Theta_{e_{1}, e_{2}}^{R}(\xi)\right)(y) \\
& =\sum_{\alpha \in r^{-1}(x)} e_{1}(y \alpha) \sigma_{\mathcal{G}}\left(y \alpha, \alpha^{-1}\right) \int_{\mathcal{G}} \overline{e_{2}\left(\gamma^{-1} u \alpha\right)} \xi\left(\gamma^{-1} u\right) \sigma_{\mathcal{G}}\left(\alpha^{-1} u^{-1} \gamma, \gamma^{-1} u\right) \mathrm{d} \nu^{r(u)}(\gamma) \\
& =\sum_{\alpha \in r^{-1}(x)} e_{1}(y \alpha) \sigma_{\mathcal{G}}\left(y \alpha, \alpha^{-1}\right) \int_{\mathcal{G}} \overline{e_{2}\left(\gamma^{-1} u \alpha\right) \sigma_{\mathcal{G}}\left(\gamma^{-1} u \alpha, \alpha^{-1}\right)} \xi\left(\gamma^{-1} u\right) \mathrm{d} \nu^{r(u)}(\gamma) \\
& =\sum_{\alpha \in r^{-1}(x)}\left(e_{1}\right)_{x}^{\alpha}(y)\left\langle\left(e_{2}\right)_{x}^{\alpha}, \xi_{x}\right\rangle_{x},
\end{aligned}
$$

where we used the 2-cocycle identity

$$
\sigma_{\mathcal{G}}\left(\alpha^{-1}, u^{-1} \gamma\right) \sigma_{\mathcal{G}}\left(\alpha^{-1} u^{-1} \gamma, \gamma^{-1} u\right)=\sigma_{\mathcal{G}}\left(\alpha^{-1}, s\left(\gamma^{-1} u\right)\right) \sigma_{\mathcal{G}}\left(u^{-1} \gamma, \gamma^{-1} u\right)=1
$$


which implies that $\sigma_{\mathcal{G}}\left(\alpha^{-1} u^{-1} \gamma, \gamma^{-1} u\right)=\overline{\sigma_{\mathcal{G}}\left(\alpha^{-1}, u^{-1} \gamma\right)}$. Then using Lemma 2.18

$$
\overline{\sigma_{\mathcal{G}}\left(\alpha^{-1}, u^{-1} \gamma\right)}=\sigma_{\mathcal{G}}\left(\gamma^{-1} u, \alpha\right)=\overline{\sigma_{\mathcal{G}}\left(\gamma^{-1} u \alpha, \alpha^{-1}\right)} .
$$

This completes the proof.

Given elements $w_{j}^{\alpha_{1}}, w_{k}^{\alpha_{2}} \in \mathfrak{h}_{x}=L^{2}\left(\mathcal{G}_{X}, \mathrm{~d} \nu^{r \circ s^{-1}(x)}\right)$, we can compute that

$$
\begin{aligned}
\left\langle w_{j}^{\alpha_{1}}, w_{k}^{\alpha_{2}}\right\rangle_{x} & =\omega_{x}\left(\delta_{\alpha_{1}}\left(w_{j} \mid w_{k}\right) \mathcal{H}_{\alpha_{2}}^{*}\right)=\left(\delta_{\alpha_{1}} *\left(w_{j} \mid w_{k}\right)_{\mathcal{H}}\right)\left(\alpha_{2}\right) \\
& =\left(w_{j} \mid w_{k}\right)_{\mathcal{H}}\left(\alpha_{1}^{-1} \alpha_{2}\right) \sigma_{\mathcal{H}}\left(\alpha_{1}, \alpha_{1}^{-1} \alpha_{2}\right) .
\end{aligned}
$$

Hence, if $\left\langle w_{j}^{\alpha_{1}}, w_{k}^{\alpha_{2}}\right\rangle_{x}=\delta_{j, k} \delta_{\alpha_{1}, \alpha_{2}}$ for some $\alpha_{1}, \alpha_{2} \in r^{-1}(x)$, the 2-cocycle term will be 1 .

At this point we can follow the same arguments as those in Theorem 3.4 and Sect. 3.3 , so we summarise our results.

Proposition 4.4 If $\left(e_{j}\right)_{j \in J}$ is a right $C^{*}$-module frame of $\mathcal{G}^{2}\left(\mathcal{G}_{X}, \sigma\right)_{\mathcal{H}}$, then for all $x \in X$ the set $\left\{\left(e_{j}\right)_{x}^{\alpha}\right\}$ for $j \in J$ and $\alpha \in r^{-1}(x)$ is a normalised tight frame of $\mathfrak{h}_{x}$.

We can again define a representation of $\pi_{x}: C_{r}^{*}\left(\mathcal{G}, \sigma_{\mathcal{G}}\right) \rightarrow \mathcal{B}\left(\mathfrak{h}_{x}\right)$, where

$$
\begin{aligned}
& \left(\pi_{x}(f) \xi_{x}\right)(y)=\int_{\mathcal{G}} f(\gamma) \xi\left(\gamma^{-1} y\right) \sigma_{\mathcal{G}}\left(\gamma, \gamma^{-1} y\right) \mathrm{d} \nu^{r \circ s^{-1}(x)}(\gamma), \\
& f \in C_{c}(\mathcal{G}), \quad \xi \in C_{c}\left(\mathcal{G}_{X}\right) .
\end{aligned}
$$

We consider the case of pre- $C^{*}$-algebras $\mathcal{S}_{k}(\mathcal{G}, \sigma) \subset C_{r}^{*}\left(\mathcal{G}, \sigma_{\mathcal{G}}\right)$ and $\mathcal{S}_{k}(\mathcal{H}, \sigma) \subset$ $C_{r}^{*}\left(\mathcal{H}, \sigma_{\mathcal{H}}\right)$ defined from families of derivations with a pre-Morita equivalence bimodule $\mathcal{S}_{k}(\mathcal{G}, \sigma) \mathcal{S}_{k}\left(\mathcal{G}_{X}, \sigma\right) \mathcal{S}_{k}(\mathcal{H}, \sigma)$ defined from a family of maps $\nabla_{j}: C_{c}(Z) \rightarrow C_{c}(Z)$ using the construction in Proposition 2.13.

Proposition 4.5 Let $k=0, \ldots, \infty$ and $p=p^{*}=p^{2} \in M_{n}\left(\mathcal{S}_{k}(\mathcal{G}, \sigma)\right)$. There are elements $\left\{v_{j}\right\}_{j=1}^{m} \subset p \mathcal{S}_{k}\left(\mathcal{G}_{X}, \sigma\right)_{\mathcal{S}_{k}(\mathcal{H}, \sigma)}^{\oplus n}$ such that for all $x \in X,\left\{v_{1}^{\beta}, \ldots, v_{m}^{\beta}\right\}_{\beta \in r^{-1}(x)}$ is a normalised tight frame of $\pi_{x}(p) \mathfrak{h}_{x}^{\oplus n}$ that is finite under the seminorms $\|\cdot\|_{l, x}$ on $\mathfrak{h}_{x}$ for $0 \leq l \leq k$. This tight frame is an orthonormal basis for all $x \in \mathcal{H}^{(0)}$ if and only if $p \mathcal{S}_{k}\left(\mathcal{G}_{X}, \sigma\right)_{\mathcal{S}_{k}(\mathcal{H}, \sigma)}^{\oplus n} \cong \mathcal{S}_{k}(\mathcal{H}, \sigma)^{\oplus m}$.

\section{Frames of Translates and Wannier Bases for the Delone Groupoid}

\subsection{Delone Sets and the Transversal Groupoid}

We review some of the material from [6] as outlined in [11]. We denote by $B(x ; K) \subset$ $\mathbb{R}^{d}$ the open ball centered at $x$ with radius $K>0$.

Definition 5.1 Let $\mathcal{L} \subset \mathbb{R}^{d}$ be discrete and infinite and fix $0<r<R$.

(1) $\mathcal{L}$ is $r$-uniformly discrete if $|B(x ; r) \cap \mathcal{L}| \leq 1$ for all $x \in \mathbb{R}^{d}$. 
(2) $\mathcal{L}$ is $R$-relatively dense if $|B(x ; R) \cap \mathcal{L}| \geq 1$ for all $x \in \mathbb{R}^{d}$.

An $r$-uniformly discrete and $R$-relatively dense set $\mathcal{L}$ is called an $(r, R)$-Delone set.

Proposition 5.2 [4, Sect. 3.2] The set of $(r, R)$-Delone sets is a compact and metrizable space. Let $d_{H}$ denote the Hausdorff distance between sets. A neighbourhood base at $\mathcal{L} \in \operatorname{Del}_{(r, R)}$ is given by the sets

$$
U_{\varepsilon, M}(\mathcal{L})=\left\{\mathcal{L}^{\prime} \in \operatorname{Del}_{(r, R)}: d_{H}\left(\mathcal{L} \cap B(0 ; M), \mathcal{L}^{\prime} \cap B(0 ; M)\right)<\varepsilon\right\}, \quad M, \varepsilon>0 .
$$

The set of Delone sets $\operatorname{Del}_{(r, R)}$ is clearly invariant under translations and rotations.

Definition 5.3 Let $\Lambda$ be a an $(r, R)$-Delone subset of $\mathbb{R}^{d}$. The continuous hull of $\Lambda$ is the dynamical system $\left(\Omega_{\Lambda}, \mathbb{R}^{d}, T\right)$, where $\Omega_{\Lambda} \subset \operatorname{Del}_{(r, R)}$ is the closure of the orbit of $\Lambda$ under the translation action.

The continuous hull of $\Lambda$ therefore gives a locally compact Hausdorff groupoid $\Omega_{\Lambda} \rtimes \mathbb{R}^{d}$. This groupoid admits a transversal in the sense of Definition 4.1.

Definition 5.4 The transversal of $\Lambda$ is given by the set

$$
\Omega_{0}=\left\{\mathcal{L} \in \Omega_{\Lambda}: 0 \in \mathcal{L}\right\}
$$

We see that $\Omega_{0}$ is a closed subset of $\Omega_{\Lambda}$ and so is compact.

Proposition 5.5 [21, Lemma 2] Given a Delone set $\Lambda$ with transversal $\Omega_{0}$, define the set

$$
\mathcal{G}_{\text {Del }}:=\left\{(\mathcal{L}, x) \in \Omega_{0} \times \mathbb{R}^{d}: x \in \mathcal{L}\right\},
$$

with maps

$$
\begin{aligned}
(\mathcal{L}, x)^{-1} & =(\mathcal{L}-x,-x), & & (\mathcal{L}, x) \cdot(\mathcal{L}-x, y)=(\mathcal{L}, x+y), \\
s(\mathcal{L}, x) & =\mathcal{L}-x, & & r(\mathcal{L}, x)=\mathcal{L}
\end{aligned}
$$

and unit space $\mathcal{G}^{(0)}=\Omega_{0}$. Then $\mathcal{G}_{\text {Del }}$ is a Hausdorff étale groupoid in the relative topology inherited from $\Omega_{0} \times \mathbb{R}^{d}$.

Notation Following the previous proposition, we will let $\mathcal{G}_{\text {Del }}$ denote the étale groupoid from a Delone set. We let $\mathcal{F}=\Omega_{\Lambda} \rtimes \mathbb{R}^{d}$ be the crossed product groupoid.

Proposition 5.6 [11, Proposition 2.16] Let $\mathcal{L} \subset \mathbb{R}^{d}$ be an $(r, R)$-Delone set with transversal $\Omega_{0}$ and associated groupoid $\mathcal{G}_{\text {Del. }}$ For $U \subset \Omega_{0}$ an open set, the sets

$V_{(U, y, \varepsilon)}:=(U \times B(y ; \varepsilon)) \cap \mathcal{G}_{\text {Del }}=\left\{(\mathcal{L}, x) \in \Omega_{0} \times \mathbb{R}^{d}: \mathcal{L} \in U, x \in \mathcal{L} \cap B(y ; \varepsilon)\right\}$,

form a base for the topology on $\mathcal{G}_{\mathrm{Del}}$. For $0<\varepsilon<r / 2$, the restriction $s: V_{(U, y, \varepsilon)} \rightarrow$ $\Omega_{0}$ is a homeomorphism onto its image. Moreover, the set $\Omega_{0} \subset \Omega_{\Lambda}$ is an abstract transversal and the groupoid $\mathcal{G}_{\text {Del }} \subset \Omega_{\Lambda} \rtimes \mathbb{R}^{d}$, with the subspace topology, is equivalent to $\Omega_{\Lambda} \rtimes \mathbb{R}^{d}$. 
Let us now fix a normalised 2-cocycle $\sigma:\left(\Omega_{\Lambda} \rtimes \mathbb{R}^{d}\right)^{(2)} \rightarrow \mathbb{T}, \sigma\left(\gamma, \gamma^{-1}\right)=1$ for all $\gamma \in \Omega_{\Lambda} \rtimes \mathbb{R}^{d}$, which also restricts to a 2-cocycle on $\mathcal{G}_{\text {Del }}$. Our main motivation to consider such twists comes from the following example.

Example 5.7 (Magnetic twists, Sect. 2.2 of [7]) Working with the continuous hull $\Omega_{\Lambda} \rtimes \mathbb{R}^{d}$, we can define a 2-cocycle, $\sigma: \mathcal{F}^{(2)} \rightarrow \mathbb{T}$ as follows. We first define a parametrised magnetic field as a continuous map $B: \Omega_{\Lambda} \rightarrow \bigwedge^{2} \mathbb{R}^{d}$. Then we define

$\sigma((\mathcal{L}, x),(\mathcal{L}-x, y))=\exp \left(-i \Gamma_{\mathcal{L}}\langle 0, x, x+y\rangle\right), \quad \Gamma_{\mathcal{L}}\langle x, y, z\rangle=\int_{\langle x, y, z\rangle} B(\mathcal{L})$

and $\langle x, y, z\rangle \subset \mathbb{R}^{2 d}$ is the triangle with corners $x, y, z \in \mathbb{R}^{d}$. Hence $\Gamma_{\mathcal{L}}\langle 0, x, x+y\rangle$ measures the magnetic flux through the triangle defined by the points $0, x, x+y \in \mathbb{R}^{d}$. It is shown in [7] that $\sigma$ is a well-defined 2-cocycle. We remark that $\sigma$ will always be trivial for $d=1$ and is normalised because

$$
\sigma((\mathcal{L}, x),(\mathcal{L}-x,-x))=\exp (-i \Gamma\langle 0, x, 0\rangle)=1
$$

If the magnetic field is constant over $\Omega_{\Lambda}$, then our general flux equation can be described using a real-valued and skew-symmetric matrix $B$ with

$$
\sigma((\mathcal{L}, x),(\mathcal{L}-x, y))=\exp (-i\langle x, B(x+y)\rangle)=\exp (-i\langle x, B y\rangle) .
$$

The 2-cocycle $\sigma$ on $\Omega_{\Lambda} \rtimes \mathbb{R}^{d}$ also restricts to a normalised 2-cocycle on $\mathcal{G}_{\text {Del }}$, where we note that if $((\mathcal{L}, x),(\mathcal{L}-x, y)) \in \mathcal{G}_{\text {Del }}^{(2)}$, the points $0, x, x+y \in \mathcal{L}$ and so $\Gamma_{\mathcal{L}}\langle 0, x, x+y\rangle$ gives a flux through the triangle with points in $\mathcal{L}$.

Remark 5.8 Given a groupoid 2-cocycle $\sigma:\left(\Omega_{\Lambda} \rtimes \mathbb{R}^{d}\right)^{(2)} \rightarrow \mathbb{T}$, the twisted groupoid $C^{*}$-algebra $C_{r}^{*}\left(\Omega_{\Lambda} \rtimes \mathbb{R}^{d}, \sigma\right)$ is canonically isomorphic to the twisted crossed product $C\left(\Omega_{\Lambda}\right) \rtimes_{\sigma^{\prime}} \mathbb{R}^{d}$, where

$$
\sigma^{\prime}: \mathbb{R}^{d} \times \mathbb{R}^{d} \rightarrow \mathcal{U}\left(C\left(\Omega_{\Lambda}\right)\right), \quad \sigma^{\prime}(x, y)=\sigma((\mathcal{L}, x),(\mathcal{L}-x, y)) .
$$

Regularity, smoothness and decay properties of functions on $\Omega_{\Lambda} \rtimes \mathbb{R}^{d}$ and $\mathcal{G}_{\text {Del }}$ are encoded via the groupoid 1-cocycles

$$
c_{k}: \Omega_{\Lambda} \rtimes \mathbb{R}^{d} \rightarrow \mathbb{R}, \quad c(\mathcal{L}, x)=x_{k}, \quad k \in\{1, \ldots, d\} .
$$

and their restrictions to $\mathcal{G}_{\text {Del }}$. It is shown in [11, Proposition 2.17] that the groupoid cycles are exact, in that $c_{k}^{-1}(0)$ has a Haar system and $c_{k}$ is a quotient map onto its image.

Given the cocycles $c_{j}: \Omega_{\Lambda} \rtimes \mathbb{R}^{d} \rightarrow \mathbb{R}^{d}$, we obtain families of commuting derivations $\left\{\partial_{j}\right\}_{j=1}^{d}$ on both $C_{c}\left(\Omega_{\Lambda} \rtimes \mathbb{R}^{d}, \sigma\right)$ and $C_{c}\left(\mathcal{G}_{\text {Del }}, \sigma\right)$ given by $\left(\partial_{j} f\right)(\mathcal{L}, x)=$ $x_{j} f(\mathcal{L}, x)$. For $k=0, \ldots, \infty$, we obtain pre- $C^{*}$-algebra completions $\mathcal{A}_{k}$ of $C_{c}\left(\Omega_{\Lambda} \rtimes \mathbb{R}^{d}, \sigma\right)$ and $\mathcal{B}_{k}$ of $C_{c}\left(\mathcal{G}_{\mathrm{Del}}, \sigma\right)$ using Proposition 2.10. 


\subsection{The Transversal $\mathcal{G}_{\text {Del }}-$ Space and Its Localisation}

Following Sect. 4, we consider the space

$$
\mathcal{F}_{\Omega_{0}}:=\left\{(\mathcal{L}, x) \in \Omega_{\Lambda} \rtimes \mathbb{R}^{d}: x \in \mathcal{L}\right\}, \quad s: \mathcal{F}_{\Omega_{0}} \rightarrow \Omega_{0}, \quad s(\mathcal{L}, x)=\mathcal{L}-x,
$$

which implements a groupoid equivalence between $\mathcal{F}=\Omega_{\Lambda} \rtimes \mathbb{R}^{d}$ and $\mathcal{G}_{\text {Del }}$. Thus $C_{c}\left(\mathcal{F}_{\Omega_{0}}\right)$ is a pre-Morita equivalence bimodule for $C_{c}\left(\Omega_{\Lambda} \rtimes \mathbb{R}^{d}, \sigma\right)$ and $C_{c}\left(\mathcal{G}_{\text {Del }}, \sigma\right)$ and can be completed into the Morita equivalence bimodule $\mathcal{F} L^{2}\left(\mathcal{F}_{\Omega_{0}}, \sigma\right)_{\mathcal{G}_{\text {Del }}}$ between $C_{r}^{*}\left(\Omega_{\Lambda} \rtimes \mathbb{R}^{d}, \sigma\right)$ and $C_{r}^{*}\left(\mathcal{G}_{\text {Del }}, \sigma\right)$.

Lemma 5.9 The restrictions of the cocycles $c_{j}: \Omega_{\Lambda} \rtimes \mathbb{R}^{d} \rightarrow \mathbb{R}$ to $\mathcal{F}_{\Omega_{0}}$ define maps

$$
\nabla_{j}: C_{c}\left(\mathcal{F}_{\Omega_{0}}\right) \rightarrow C_{c}\left(\mathcal{F}_{\Omega_{0}}\right), \quad \nabla_{j}(f)(\mathcal{L}, x):=x_{j} f(\mathcal{L}, x)
$$

For all $a \in C_{c}(\mathcal{F}, \sigma), b \in C_{c}\left(\mathcal{G}_{\text {Del }}, \sigma\right)$ and $\xi, \eta \in C_{c}\left(\mathcal{F}_{\Omega_{0}}\right)$ we have

$$
\begin{aligned}
\nabla_{j}(a \cdot \xi \cdot b) & =\partial_{j}(a) \cdot \xi \cdot b+a \cdot \nabla_{j}(\xi) \cdot b+a \cdot \xi \cdot \partial_{j}(b) \\
\partial_{j}(\xi \mid \eta)_{\mathcal{G}_{\text {Del }}} & =\left(\xi \mid \nabla_{j}(\eta)\right)_{\mathcal{G}_{\text {Del }}}-\left(\nabla_{j}(\xi) \mid \eta\right)_{\mathcal{G}_{\text {Del }}}
\end{aligned}
$$

Consequently, for all $k=0, \ldots, \infty$, the space $C_{c}\left(\mathcal{F}_{\Omega_{0}}\right)$ can be completed into a pre-Morita equivalence bimodule $\mathcal{A}_{k} \mathcal{S}_{k}\left(\mathcal{F}_{\Omega_{0}}, \sigma\right)_{\mathcal{B}_{k}}$ for the pre-C ${ }^{*}$-algebras $\mathcal{A}_{k} \subset$ $C_{r}^{*}\left(\Omega_{\Lambda} \rtimes \mathbb{R}^{d}, \sigma\right)$ and $\mathcal{B}_{k} \subset C_{r}^{*}\left(\mathcal{G}_{\mathrm{Del}}, \sigma\right)$.

Proof As $\mathcal{F}_{\Omega_{0}}$ and $\mathcal{G}_{\text {Del }}$ are subspaces of $\Omega_{\Lambda} \rtimes \mathbb{R}^{d}$ and the bimodule structure and inner product are induced by the convolution product in $C_{c}\left(\Omega_{\Lambda} \rtimes \mathbb{R}^{d}, \sigma\right)$, the required identities follow from the fact that multiplication by $x_{j}$ is a derivation of $C_{c}\left(\Omega_{\Lambda} \rtimes\right.$ $\left.\mathbb{R}^{d}, \sigma\right)$. The result now follows from Propositions 2.10 and 2.13.

For every $\mathcal{L} \in \Omega_{0}$, there is a state $\omega_{\mathcal{L}}$ on $C_{r}^{*}\left(\mathcal{G}_{\text {Del }}, \sigma\right)$ such that $\omega_{\mathcal{L}}(f)=f(\mathcal{L}, 0)$ for $f \in C_{c}\left(\mathcal{G}_{\text {Del }}, \sigma\right)$. Note that

$$
\omega_{\mathcal{L}}\left(f^{*} f\right)=\sum_{y \in \mathcal{L}}|f(\mathcal{L}-y,-y)|^{2}, \quad \omega_{\mathcal{L}}\left(1_{C_{r}^{*}\left(\mathcal{G}_{\text {Del }}\right)}\right)=1
$$

and so $\omega_{\mathcal{L}}$ is faithful. From this point, all results from Sect. 4 apply to the Delone groupoid setting. Though for concreteness, we highlight some key aspects of this example.

Lemma 5.10 For every $\mathcal{L} \in \Omega_{0}$, the localised Hilbert space $\mathfrak{h}_{\mathcal{L}} \cong L^{2}\left(\mathbb{R}^{d}\right)$.

Proof We define a map $\beta_{\mathcal{L}} \rightarrow L^{2}\left(\mathbb{R}^{d}\right)$ that agrees with the localised Hilbert space inner product. Namely, we consider $\beta_{\mathcal{L}}: L^{2}\left(\mathcal{F}_{\Omega_{0}}\right) \rightarrow L^{2}\left(\mathbb{R}^{d}\right)$, given by $\left[\beta_{\mathcal{L}}(\xi)\right](x)=$ $\xi(\mathcal{L}-x,-x)$ for almost all $x$. To see why this is true, we first note that for any $\mathcal{L} \in \Omega_{0}$, $s^{-1}(\mathcal{L})=\{(\mathcal{L}-x,-x)\}_{x \in \mathbb{R}^{d}}$ and the measure on $s^{-1}(\mathcal{L})$ is just the Lebesgue measure on $\mathbb{R}^{d}$. We also see that

$$
\left\langle\xi_{1}, \xi_{2}\right\rangle_{\mathcal{L}}=\left(\xi_{1} \mid \xi_{2}\right)_{C_{r}^{*}\left(\mathcal{G}_{\text {Del }}\right)}(\mathcal{L}, 0)=\int_{\mathbb{R}^{d}} \overline{\xi_{1}(\mathcal{L}-y,-y)} \xi_{2}(\mathcal{L}-y,-y) \mathrm{d} y .
$$


Hence we see there is a canonical identification of $\mathfrak{h}_{\mathcal{L}}$ with $\beta_{\mathcal{L}}\left[L^{2}\left(\mathcal{F}_{\Omega_{0}}\right)\right] \cong L^{2}\left(\mathbb{R}^{d}\right)$.

For any $\xi \in \mathcal{F}^{2} L^{2}\left(\mathcal{F}_{\Omega_{0}}\right)_{\mathcal{G}_{\text {Del }}}$, let $\xi_{\mathcal{L}} \in \mathfrak{h}_{\mathcal{L}}$ be its localisation. Because $\nabla^{\alpha} \xi(\mathcal{L}, x)=$ $x^{\alpha} \xi(\mathcal{L}, x)$ for $\alpha \in \mathbb{N}^{d}$ and $\xi \in C_{c}\left(\mathcal{F}_{\Omega_{0}}\right)$, Proposition 2.13 gives the following.

Lemma 5.11 For any $k=0, \ldots, \infty$ and $\mathcal{L} \in \Omega_{0}$, every element in dense subspace $\beta_{\mathcal{L}}\left[\mathcal{S}_{k}\left(\mathcal{F}_{\Omega_{0}}\right)\right] \subset \mathfrak{h}_{\mathcal{L}}$ has polynomial decay of at least degree $k$,

$$
\left\|x^{\alpha} \xi_{\mathcal{L}}\right\|_{\mathfrak{h}_{\mathcal{L}}} \leq C, \quad \xi \in \mathcal{S}_{k}\left(\mathcal{F}_{\Omega_{0}}\right), \alpha \in \mathbb{N}^{d},|\alpha| \leq k
$$

Lemma 5.12 Let $\chi$ be a smooth and real-valued bump-function such that $\operatorname{supp}(\chi) \subset$ $B(0 ; r / 2), \chi(x)=\chi(-x), \chi(0)=1$ and $\|\chi\|_{2}=1$.

(i) Extend $\chi$ to a function $\chi \in C_{c}\left(\mathcal{F}_{\Omega_{0}}\right)$ such that $\chi(\mathcal{L}, x)=\chi(x)$. Then $(\chi \mid$ $\chi)_{\mathcal{G}_{\text {Del }}}=1_{\mathcal{G}_{\text {Del }}}$.

(ii) Given $p \in \mathbb{R}^{d}$, define the function $\chi_{p} \in C_{c}\left(\mathcal{G}_{\text {Del }}, \sigma\right)$ by $\chi_{p}(\mathcal{L}, x)=\chi(x-p)$. Then for any $\mathcal{L} \in \Omega_{0}$ and $p, q \in \mathcal{L}, \omega_{\mathcal{L}}\left(\chi_{p} * \chi_{q}^{*}\right)=\delta_{p, q}$.

Proof For part (i) we compute

$$
(\chi \mid \chi)_{\mathcal{G}_{\text {Del }}}(\mathcal{L}, y)=\int_{\mathbb{R}^{d}} \chi(\mathcal{L}-z,-z) \chi(\mathcal{L}-z, y-z) \sigma((\mathcal{L}, z),(\mathcal{L}-z, y-z)) \mathrm{d} z .
$$

The integral will be zero unless $-z, y-z \in \mathcal{L}-z \cap B(0 ; r / 2)$. But because $y \in \mathcal{L}$, $\mathcal{L} \in \Omega_{0}$ and $\mathcal{L}$ is uniformly $r$-discrete, this will only happen when $y=0$. Then, because the 2-cocycle is 1 when $y=0$ and $\|\chi\|_{2}=1,(\chi \mid \chi)_{\mathcal{G}_{\text {Del }}}(\mathcal{L}, y)=\delta_{y, 0}=1_{\mathcal{G}_{\text {Del }}}(\mathcal{L}, y)$.

For part (ii) we compute that

$$
\begin{aligned}
\left(\chi_{p} * \chi_{q}^{*}\right)(\mathcal{L}, 0) & =\sum_{y \in \mathcal{L}} \chi_{p}(\mathcal{L}, y) \overline{\chi_{q}(\mathcal{L}, y)} \sigma((\mathcal{L}, y),(\mathcal{L}-y,-y)) \\
& =\sum_{y \in \mathcal{L}} \chi(y-p) \chi(y-q)=\chi(p-q)=\delta_{p, q}
\end{aligned}
$$

where we have used that $\operatorname{supp}(\chi) \subset B(0, r / 2)$ and $\mathcal{L}$ is $r$-discrete.

Given $(\mathcal{L}, y) \in \mathcal{G}_{\text {Del }}$, define the action

$\xi^{(\mathcal{L}, y)}(x)=\xi(\mathcal{L}-x, y-x) \sigma((\mathcal{L}-x, y-x),(\mathcal{L}-y,-y)) \in \mathfrak{h}_{\mathcal{L}}, \quad \xi \in L^{2}\left(\mathcal{F}_{\Omega_{0}}\right)$.

In the case that $\sigma$ is comes from a magnetic twist that is constant over the unit space $\Omega_{\Lambda}$, we see that $\xi^{(\mathcal{L}, y)}(x)=e^{-i\langle x, B y\rangle} \xi(\mathcal{L}-x, y-x)$ with $B$ a real-valued skew-adjoint matrix describing the magnetic field. Lemmas 4.3 and 5.12 now give the following.

Lemma 5.13 (i) Recall the functions $\left\{\chi_{p}\right\}$ from Lemma 5.12. Then for any $\xi \in$ $L^{2}\left(\mathcal{F}_{\Omega_{0}}\right)$ and $(\mathcal{L}, p) \in \mathcal{G}_{\text {Del }}, \xi^{(\mathcal{L}, p)}=\left(\xi \cdot \chi_{p}^{*}\right)_{\mathcal{L}}$.

(ii) Let $e \in \mathcal{F} L^{2}\left(\mathcal{F}_{\Omega_{0}}, \sigma\right)_{\mathcal{G}_{\text {Del }}}$ be such that $(e \mid e)_{C_{r}^{*}\left(\mathcal{G}_{\text {Del }}\right)}=1_{C_{r}^{*}\left(\mathcal{G}_{\text {Del }}\right)}$. Then for any $\mathcal{L} \in \Omega_{0}$ the set $\left\{e^{(\mathcal{L}, y)}\right\}_{y \in \mathcal{L}}$ is an orthonormal system in $\mathfrak{h}_{\mathcal{L}}$. 
Proposition 5.14 Let $\left(e_{j}\right)_{j \in J}$ be a (countable) right frame of $\mathcal{F} L^{2}\left(\mathcal{F}_{\Omega_{0}}, \sigma\right)_{\mathcal{G}_{\text {Del }}}$. Then for all $\mathcal{L} \in \Omega_{0}$, the set $\left\{e_{j}^{(\mathcal{L}, y)}\right\}_{j \in J, y \in \mathcal{L}}$ is a normalised tight frame for $\mathfrak{h}_{\mathcal{L}} \cong L^{2}\left(\mathbb{R}^{d}\right)$. If $\left(e_{j}\right)_{j \in J} \subset \mathcal{A}_{k} \mathcal{S}_{k}\left(\mathcal{F}_{\Omega_{0}}, \sigma\right)_{\mathcal{B}_{k}}$ for $k=0, \ldots, \infty$, then the normalised tight frame $\left\{e_{j}^{(\mathcal{L}, y)}\right\}$ has polynomial decay of at least order $k$.

Proof The first statement is a special case of Proposition 4.4. Lemma 5.11 ensures that the elements $e_{j}^{(\mathcal{L}, 0)}(x)=e_{j}(\mathcal{L}-x,-x)$ have polynomial decay of at least degree $k$. The translation $e_{j}^{(\mathcal{L}, y)}(x)=e_{j}(\mathcal{L}-x, y-x) \sigma((\mathcal{L}-x, y-x),(\mathcal{L}-y,-y))$ will have the same decay properties for all $y \in \mathcal{L}$.

We can define an action $\pi_{\mathcal{L}}: C\left(\Omega_{\Lambda}\right) \rtimes \mathbb{R}^{d}$ on the localisation Hilbert space $\mathfrak{h}_{\mathcal{L}} \cong L^{2}\left(\mathbb{R}^{d}\right)$ by

$$
\left(\pi_{\mathcal{L}}(f) \xi_{\mathcal{L}}\right)(x)=(f \cdot \xi)_{\mathcal{L}}(x)=(f \cdot \xi)(\mathcal{L}-x,-x) .
$$

Explicitly, we can compute that

$$
(f \cdot \xi)(\mathcal{L}-x,-x)=\int_{\mathbb{R}^{d}} f(\mathcal{L}-x, u-x) \xi_{\mathcal{L}}(u) \sigma((\mathcal{L}-x, u-x),(\mathcal{L}-u,-u)) \mathrm{d} u .
$$

Let us now consider the localisation $\pi_{\mathcal{L}}(p) \mathfrak{h}_{\mathcal{L}}^{\oplus n}$ for $p \in M_{n}\left(C_{r}^{*}\left(\Omega_{\Lambda} \rtimes \mathbb{R}^{d}, \sigma\right)\right)$ a projection.

Theorem 5.15 Let $k=0, \ldots, \infty$ and $p=p^{*}=p^{2} \in M_{n}\left(\mathcal{A}_{k}\right)$. Then there are elements $\left\{e_{j}\right\}_{j=1}^{m} \subset p \mathcal{S}_{k}\left(\mathcal{F}_{\Omega_{0}}, \sigma\right)_{\mathcal{B}_{k}}^{\oplus n}$ such that for all $\mathcal{L} \in \Omega_{0},\left\{e_{1}^{(\mathcal{L}, y)}, \ldots, e_{m}^{(\mathcal{L}, y)}\right\}_{y \in \mathcal{L}}$ is a normalised tight frame of $\pi_{\mathcal{L}}(p) L^{2}\left(\mathbb{R}^{d}, \mathbb{C}^{n}\right)$ with polynomial decay of at least degree $k$. The normalised tight frame $\left\{e_{1}^{(\mathcal{L}, y)}, \ldots, e_{m}^{(\mathcal{L}, y)}\right\}_{y \in \mathcal{L}}$ is an orthonormal basis for all $\mathcal{L} \in \Omega_{0}$ if and only if $p \mathcal{S}_{k}\left(\mathcal{F}_{\Omega_{0}}, \sigma\right)_{\mathcal{B}_{k}}^{\oplus n} \cong \mathcal{B}_{k}^{\oplus m}$.

For concreteness, we note that the case $p \in M_{n}(\mathcal{A})$ with $\mathcal{A}=\mathcal{A}_{\infty}$ gives a normalised tight frame with faster than polynomial decay.

Remark 5.16 (Invariance under homotopies of 2-cocycles) Let us briefly consider the stability of Theorem 5.15 under deformations of the groupoid 2-cocycle $\sigma$ using results from Gillaspy [17]. Given the groupoid $\mathcal{F}=\Omega_{\Lambda} \rtimes \mathbb{R}^{d}$, we can consider the trivial bundle of groupoids $\mathcal{F} \times[0,1]$ equipped with the product topology so that groupoid operations preserve the fibres and such that $\mathcal{F} \times[0,1]$ is a locally compact Hausdorff groupoid. A homotopy of groupoid 2-cocycles is a groupoid 2-cocycle $\omega:(\mathcal{F} \times[0,1])^{(2)} \rightarrow \mathbb{T}$, which will give rise to a family of 2 -cocycles $\left\{\omega_{t}\right\}_{t \in[0,1]}$ on $\mathcal{F}$ that is continuous in $t$.

Because $\Omega_{\Lambda} \rtimes \mathbb{R}^{d}$ satisfies the Baum-Connes conjecture with coefficients, [17, Theorem 5.1] applies, which says that the evaluation map

$$
q_{t}: C_{r}^{*}\left(\left(\Omega_{\Lambda} \rtimes \mathbb{R}^{d}\right) \times[0,1], \omega\right) \rightarrow C_{r}^{*}\left(\Omega_{\Lambda} \rtimes \mathbb{R}^{d}, \omega_{t}\right)
$$

induces an isomorphism of $K$-theory groups. Composing this isomorphism with the Morita equivalence of $\Omega_{\Lambda} \rtimes \mathbb{R}^{d}$ with $\mathcal{G}_{\text {Del }}$, given a homotopy of 2-cocycles $\sigma_{\bullet}$ on 
$\mathcal{F} \times[0,1]$ (and so on $\mathcal{G}_{\text {Del }} \times[0,1]$ by restriction), we can consider finitely generated and projective modules $P_{\bullet}$ over $C_{r}^{*}\left(\mathcal{G}_{\text {Del }} \times[0,1], \sigma_{\bullet}\right)$. Composing with the evaluation map, $P_{0}$ is a free $C_{r}^{*}\left(\mathcal{G}_{\text {Del }}, \sigma_{0}\right)$-module if and only if $P_{1}$ is a free $C_{r}^{*}\left(\mathcal{G}_{\text {Del }}, \sigma_{1}\right)$-module.

Considering the magnetic twists of Example 5.7, we can easily construct homotopies of 2-cocycles via a continuous map $B_{\bullet}: \Omega_{\Lambda} \times[0,1] \rightarrow \bigwedge^{2} \mathbb{R}^{d}$ which restricts to a continuous path $\left\{B_{t}\right\}_{t \in[0,1]}$ of magnetic fields.

\subsection{The Localisation Dichotomy}

Decay properties of the normalised tight frame in Theorem 5.15 come from the seminorms on the dense submodules $\mathcal{S}_{k}\left(\mathcal{F}_{\Omega_{0}}, \sigma\right)$ over the pre- $C^{*}$-algebras $\mathcal{A}_{k} \subset$ $C_{r}^{*}\left(\Omega_{\Lambda} \rtimes \mathbb{R}^{d}, \sigma\right)$ and $\mathcal{B}_{k} \subset C_{r}^{*}\left(\mathcal{G}_{\text {Del }}, \sigma\right)$ for $k=0, \ldots, \infty$. Because each $\mathcal{A}_{k}$ is a pre- $C^{*}$-algebra, given $p=p^{*}=p^{2} \in M_{n}\left(C_{r}^{*}\left(\Omega_{\Lambda} \rtimes \mathbb{R}^{d}, \sigma\right)\right)$ and $0<\varepsilon<1$, there is some $p_{k}=p_{k}^{*}=p_{k}^{2} \in M_{n}\left(\mathcal{A}_{k}\right) \subset M_{n}\left(C_{r}^{*}\left(\Omega_{\Lambda} \rtimes \mathbb{R}^{d}, \sigma\right)\right)$ such that $\left\|p-p_{k}\right\|<\varepsilon$ in $C^{*}$-norm as well as a unitary $u_{k}$ in $M_{n}\left(C_{r}^{*}\left(\Omega_{\Lambda} \rtimes \mathbb{R}^{d}, \sigma\right)\right)$ such that $p_{k}=u_{k}^{*} p u_{k}$ (see [9, Sect. 4]). Consequently the finitely generated and projective $C^{*}\left(\mathcal{G}_{\text {Del }}, \sigma\right)$-modules $p L^{2}\left(\mathcal{F}_{\Omega_{0}}, \sigma\right)_{\mathcal{G}_{\text {Del }}}^{\oplus n}$ and $p_{k} L^{2}\left(\mathcal{F}_{\Omega_{0}}, \sigma\right)_{\mathcal{G}_{\text {Del }}}^{\oplus n}$ are isomorphic. The module $p_{k} L^{2}\left(\mathcal{F}_{\Omega_{0}}, \sigma\right)_{\mathcal{G}_{\text {Del }}}^{\oplus n}$ contains the dense submodule $p_{k} \mathcal{S}_{k}\left(\mathcal{F}_{\Omega_{0}}, \sigma\right)_{\mathcal{B}_{k}}^{\oplus n}$. Note that we can choose $p_{k}=p_{\infty}$ for all $k \geq 1$.

By comparing $C^{*}$-module frames for $\mathcal{S}_{\infty}\left(\mathcal{F}_{\Omega_{0}}, \sigma\right) \subset \mathcal{S}_{1}\left(\mathcal{F}_{\Omega_{0}}, \sigma\right) \subset L^{2}\left(\mathcal{F}_{\Omega_{0}}, \sigma\right)$, we can prove a weak version of the localisation dichotomy considered in [29, Sect. 5 (arXiv version)].

Proposition 5.17 (Weak localisation dichotomy) Let $p=p^{*}=p^{2} \in M_{n}\left(C_{r}^{*}\left(\Omega_{\Lambda} \rtimes\right.\right.$ $\left.\left.\mathbb{R}^{d}, \sigma\right)\right)$ and $p_{k}=p_{k}^{*}=p_{k}^{2} \in M_{n}\left(\mathcal{A}_{k}\right)$ be equivalent projections as above. The following statements are equivalent.

(i) There is a $C^{*}$-module isomorphism $p L^{2}\left(\mathcal{F}_{\Omega_{0}}, \sigma\right)_{\mathcal{G}_{\text {Del }}}^{\oplus n} \cong C_{r}^{*}\left(\mathcal{G}_{\mathrm{Del}}, \sigma\right)^{\oplus m}$.

(ii) There are elements $\left\{w_{j}\right\}_{j=1}^{m} \subset p_{1} \mathcal{S}_{1}\left(\mathcal{F}_{\Omega_{0}}, \sigma\right)_{\mathcal{B}_{1}}^{\oplus n}$ such that for all $\mathcal{L} \in \Omega_{0}$, the collection $\left\{w_{1}^{(\mathcal{L}, y)}, \ldots, w_{m}^{(\mathcal{L}, y)}\right\}_{y \in \mathcal{L}}$ is an orthonormal basis of $\pi_{\mathcal{L}}(p) L^{2}\left(\mathbb{R}^{d}, \mathbb{C}^{n}\right)$ and for all $y \in \mathcal{L}$,

$$
\sum_{j=1}^{m} \int_{\mathbb{R}^{d}}\left(1+|x-y|^{2}\right)\left|w_{j}^{(\mathcal{L}, y)}(x)\right|^{2} \mathrm{~d} x<\infty .
$$

(iii) There exists $\left\{e_{j}\right\}_{j=1}^{m} \subset p_{\infty} \mathcal{S}_{\infty}\left(\mathcal{F}_{\Omega_{0}}, \sigma\right)_{\mathcal{B}_{\infty}}^{\oplus n}$ where for all $\mathcal{L} \in \Omega_{0},\left\{e_{1}^{(\mathcal{L}, y)}, \ldots\right.$, $\left.e_{m}^{(\mathcal{L}, y)}\right\}_{y \in \mathcal{L}}$ is an orthonormal basis of $\pi_{\mathcal{L}}(p) L^{2}\left(\mathbb{R}^{d}, \mathbb{C}^{n}\right)$ with faster than polynomial decay.

Proof All statements except for Eq. (8) immediately follow from Theorem 5.15. To see Eq. (8), we note that the frame elements are such that $\left\|w_{j} \cdot \chi_{p}^{*}\right\|_{1}<\infty$ for $j \in\{1, \ldots, m\}, p \in \mathbb{R}^{d}$ and $\|\cdot\|_{1}$ the seminorm on $\mathcal{S}_{1}\left(\mathcal{F}_{\Omega_{0}}, \sigma\right)$,

$$
\|\xi\|_{1}=\|\xi\|+\sum_{l=1}^{d}\left\|\nabla_{l} \xi\right\|
$$


Passing to the localisation, the functions $w_{j}^{(\mathcal{L}, y)}$ and $\left[\nabla_{l}\left(w_{j} \cdot \chi_{y}^{*}\right)\right]_{\mathcal{L}}(x)=\left(x_{l}-\right.$ $\left.y_{l}\right) w_{j}^{(\mathcal{L}, y)}(x)$ are in $\pi_{\mathcal{L}}(p) L^{2}\left(\mathbb{R}^{d}, \mathbb{C}^{n}\right)$ for any $y \in \mathcal{L}, j \in\{1, \ldots, m\}$ and $l \in$ $\{1, \ldots, d\}$. We can combine these cases to obtain Eq. (8).

Proposition 5.17 should be compared to the Localisation Dichotomy Conjecture in [29, Sect. 5 (arXiv version)]. We have shown that an $s_{*}$-localised Wannier basis for $s_{*}=1$ is equivalent to a Wannier basis with faster than polynomial decay, which in turn is equivalent to a free finitely generated and projective module. To improve condition (iii) to an exponentially localised Wannier basis will require more analytic arguments that fall outside the framework of pre- $C^{*}$-algebras we have considered. In Sect. 5.5, we show that conditions (i)-(iii) of Proposition 5.17 imply that the (even) noncommutative Chern numbers vanish.

Since the submission of this manuscript, the preprints [25,30] have appeared that further develop the Localisation Dichotomy Conjecture for generalised Wannier bases in dimension 2. In particular, [25, Theorem 1] shows that for gapped spectral projections of a magnetic Schrödinger operator on $L^{2}\left(\mathbb{R}^{2}\right)$ (with mild regularity assumptions), an exponentially localised Wannier basis is equivalent to an $s_{*}$-localised Wannier basis with $s_{*}>5 / 2$. Hence, for projections $p \in C_{r}^{*}\left(\Omega_{\Lambda} \rtimes \mathbb{R}^{2}, \sigma\right)$ that fall into the framework of [25], we can improve condition (iii) of Proposition 5.17 to exponential decay. The magnetic Schrödinger operator with Delone atomic potential we consider below (see Equation (9)) satisfies the regularity assumptions of [25] when $d=2$.

Remark 5.18 (Connections to the Balian-Low Theorem) Theorem 5.15 and Proposition 5.17 plays a similar role to the Balian-Low Theorem in time-frequency analysis. Brieflly, the theorem states that if a Gabor system $\left\{e^{2 \pi i m t} g(t-n)\right\}_{m, n \in \mathbb{Z}}$ forms an orthonormal basis of $L^{2}(\mathbb{R})$, then either $g$ or the Fourier transform $\hat{g}$ is such that the sum in Equation (8) with $m=1$ diverges. By the work of Luef [28], the Balian-Low Theorem can also be interpreted using finitely generated and projective modules over $C^{*}\left(\mathbb{Z}^{2}\right)$ and the fact that $C\left(\mathbb{T}^{2}\right)$ has no non-trivial projections. See [28] for more details and a generalisation to the rotation algebra $A_{\theta} \simeq C^{*}\left(\mathbb{Z}^{2}, \theta\right)$.

\subsection{Wannier Bases for Hamiltonians on $L^{2}\left(\mathbb{R}^{d}, \mathbb{C}^{n}\right)$ with Delone Potentials}

We model a particle in $\mathbb{R}^{d}$ subject to a uniform magnetic field perpendicular to the sample. We take a magnetic potential $A=\left(A_{1}, \ldots, A_{d}\right)$ such that $A_{j} \in L_{\text {loc. }}^{2}\left(\mathbb{R}^{d}\right)$ and differentiable with

$$
\frac{\partial}{\partial x_{j}} A_{k}-\frac{\partial}{\partial x_{k}} A_{j}=\text { const. }
$$

for all $j, k \in\{1, \ldots, d\}$. For simplicity, we consider constant magnetic field strength but more general fields are possible (cf. Example 5.7). The magnetic Schrödinger operator is given by 


$$
H_{0}=\sum_{j=1}^{d}\left(-i \frac{\partial}{\partial x_{j}}-A_{j}\right)^{2}
$$

We choose the symmetric gauge and define $A_{j}=-\frac{1}{2} \sum_{k=1}^{d} \theta_{j, k} x_{k}$ for $j=1, \ldots, d$, where $\theta_{j, k}$ is antisymmetric and real. Our choice of gauge gives the magnetic translations $\left\{U_{a}\right\}_{a \in \mathbb{R}^{d}}$, where for any $a \in \mathbb{R}^{d}$,

$$
\left[H_{0}, U_{a}\right]=0, \quad\left(U_{a} \psi\right)(x)=e^{-i\langle x, \theta a\rangle} \psi(x-a), \quad \psi \in L^{2}\left(\mathbb{R}^{d}\right) .
$$

Given a compact space $\Omega$ with action $T: \mathbb{R}^{d} \rightarrow \operatorname{Homeo}(\Omega)$, we can define the groupoid 2-cocycle

$$
\theta:\left(\Omega \rtimes \mathbb{R}^{d}\right)^{(2)} \rightarrow \mathbb{T}, \quad \theta\left((\omega, x),\left(T_{-x} \omega, y\right)\right)=e^{-i\langle x, \theta y\rangle},
$$

which is normalised, $\theta\left((\omega, x),\left(T_{-x} \omega,-x\right)\right)=1$, and constant over the unit space.

We wish to relate spectral properties of aperiodic Schrödinger operators to the Delone groupoid. We do this by considering atomic potentials on point sets,

$$
H_{\Gamma}=H_{0}+\sum_{p \in \Gamma} v(\cdot-p), \quad H_{0}=\sum_{j=1}^{d}\left(-i \frac{\partial}{\partial x_{j}}-A_{j}\right)^{2},
$$

where $v$ an atomic potential function. Provided the potential $V_{\Gamma}=\sum_{p \in \Gamma} v(\cdot-p)$ is essentially bounded, real valued and measurable, $H_{\Gamma}$ is essentially self-adjoint on the dense core $C_{c}^{\infty}\left(\mathbb{R}^{d}\right)$. We assume $\Gamma$ is $r$-discrete and restrict our potentials to the $K$-subharmonic functions on $\mathbb{R}^{d}$,

$$
L_{K, r}^{1}\left(\mathbb{R}^{d}\right)=\left\{f \in L^{1}\left(\mathbb{R}^{d}\right):|f(x)| \leq K r^{-d} \int_{|x-y|<r / 2}|f(y)| \text { dy for a.e. } x\right\} .
$$

Theorem 5.19 [5] Let $\Lambda$ be an $(r, R)$-Delone set and $v \in L_{K, r}^{1}\left(\mathbb{R}^{d}\right)$ be a uniformly continuous and $\mathbb{R}$-valued atomic potential. Then the family of operators $h=\left\{H_{\mathcal{L}}\right\}_{\mathcal{L} \in \Omega_{\Lambda}}$ with $H_{\mathcal{L}}$ as in Eq. (9) is affiliated to the groupoid $C^{*}$-algebra $C_{r}^{*}\left(\Omega_{\Lambda} \rtimes \mathbb{R}^{d}, \theta\right)$.

Remark 5.20 Theorem 5.19 is proved by showing that $h=\left\{H_{\mathcal{L}}\right\}_{\mathcal{L} \in \Omega_{\Lambda}}$ is affiliated to a crossed product groupoid constructed from the continuous hull of the potential $V_{\Lambda}=\sum_{p \in \Lambda} v(\cdot-p)$. In general the continuous hull of a Delone set is topologically semi-conjugate to the continuous hull of a Delone potential via a surjective map $\Omega_{\Lambda} \rightarrow \Omega_{V_{\Lambda}}$. If $\operatorname{supp}(v) \subset B\left(0, r_{v}\right)$ for some $r_{v} \leq r$, then the map is injective, see [6, Sect. 2.7].

Let us now fix an $(r, R)$-Delone set $\Lambda$ and Hamiltonian of the form Eq. (9) such that the family of Schrödinger operators $h=\left\{H_{\mathcal{L}}\right\}_{\mathcal{L} \in \Omega_{\Lambda}}$ is affiliated to the twisted 
groupoid $C^{*}$-algebra $C_{r}^{*}\left(\Omega_{\Lambda} \rtimes \mathbb{R}^{d}, \theta\right)$. With the choice of twist $\theta$, we see that for $\pi_{\mathcal{L}}: C_{r}^{*}\left(\Omega_{\Lambda} \rtimes \mathbb{R}^{d}, \theta\right) \rightarrow \mathcal{B}\left[L^{2}\left(\mathbb{R}^{d}\right)\right]$,

$\left(\pi_{\mathcal{L}}(f) \psi\right)(x)=\int_{\mathbb{R}^{d}} e^{-i\langle x, \theta u\rangle} f(\mathcal{L}-x, u-x) \psi(u) \mathrm{d} u, \quad U_{a} \pi_{\mathcal{L}}(f) U_{a}^{*}=\pi_{\mathcal{L}-a}(f)$.

The twisted groupoid algebra $C_{r}^{*}\left(\Omega_{\Lambda} \rtimes \mathbb{R}^{d}, \theta\right)$ is Morita equivalent to $C_{r}^{*}\left(\mathcal{G}_{\text {Del }}, \theta\right)$ and for every $(\mathcal{L}, y) \in \mathcal{G}_{\text {Del }}$, we have a twisted translation action

$$
\psi^{(\mathcal{L}, y)}(x)=e^{-i\langle x, B y\rangle} \psi(x-y)=\left(U_{y} \psi\right)(x) .
$$

Hence, the discrete groupoid translations are just the magnetic translations restricted to $\mathcal{L} \in \Omega_{0}$.

Recall the pre- $C^{*}$-algebra $\mathcal{A}=\mathcal{A}_{\infty} \subset C_{r}^{*}\left(\Omega_{\Lambda} \rtimes \mathbb{R}^{d}, \theta\right)$ that comes from the Fréchet completion of $C_{c}\left(\Omega_{\Lambda} \times \mathbb{R}^{d}, \theta\right)$ in the seminorms defined from the derivations $\left\{\partial_{j}\right\}_{j=1}^{d}$ (Proposition 2.10).

Lemma 5.21 Let $h$ be a self-adjoint element affiliated to $M_{n}\left(C_{r}^{*}\left(\Omega_{\Lambda} \rtimes \mathbb{R}^{d}, \theta\right)\right)$. Suppose that $\Delta \subset \sigma(h)$ is a bounded spectral region separated from $\sigma(h) \backslash \Delta$ with positive distance. Then $p_{\Delta}(h)=\chi_{\Delta}(h) \in M_{n}(\mathcal{A})$.

Proof Because $\Delta$ is an isolated spectral region, $p_{\Delta}(h)$ can be approximated arbitrarily well by $\varphi(h)$ with $\varphi \in C_{c}^{\infty}(\mathbb{R})$ such that $\varphi(x)=1$ for $x \in \Delta$ and $\varphi(x)=0$ for $x \in \sigma(h) \backslash \Delta$. Hence $\varphi(h) \in M_{n}(\mathcal{A})$.

Hence, we can adapt Theorem 5.15 to the case of Schrödinger operators on $L^{2}\left(\mathbb{R}^{d}, \mathbb{C}^{n}\right)$ with Delone atomic potentials.

Theorem 5.22 Let $\Lambda$ be a $(r, R)$-Delone set and let $H_{\Lambda}$ be a magnetic Schrödinger operator on $L^{2}\left(\mathbb{R}^{d}, \mathbb{C}^{n}\right)$ with Delone atomic potential as in $E q$. (9) with $v \in L_{K, r}^{1}\left(\mathbb{R}^{d}\right)$ and uniformly continuous. Suppose that $\Delta$ is an isolated and bounded spectral region of $\sigma\left(H_{\mathcal{L}}\right)$ for all $\mathcal{L} \in \Omega_{\Lambda}$. Then there are elements $w_{1}, \ldots, w_{m} \in p_{\Delta} \mathcal{S}_{\infty}\left(\mathcal{F}_{\Omega_{0}}, \theta\right)^{\oplus n}$ such that for all $\mathcal{L} \in \Omega_{0}$ the magnetic translates $\left\{U_{y} w_{1}^{\mathcal{L}}, \ldots, U_{y} w_{m}^{\mathcal{L}}\right\}_{y \in \mathcal{L}}$ give a normalised tight frame of $p_{\Delta}\left(H_{\mathcal{L}}\right) L^{2}\left(\mathbb{R}^{d}, \mathbb{C}^{n}\right)$ with faster than polynomial decay.

The frame $\left\{U_{y} w_{1}^{\mathcal{L}}, \ldots, U_{y} w_{m}^{\mathcal{L}}\right\}_{y \in \mathcal{L}}$ is an orthonormal basis of $p_{\Delta}\left(H_{\mathcal{L}}\right) L^{2}\left(\mathbb{R}^{d}, \mathbb{C}^{n}\right)$ for all $\mathcal{L} \in \Omega_{0}$ if and only if the finitely generated and projective $C^{*}$-module $p_{\Delta} L^{2}\left(\mathcal{F}_{\Omega_{0}}, \theta\right)_{\mathcal{G}_{\text {Del }} n}^{\oplus n} \cong C_{r}^{*}\left(\mathcal{G}_{\text {Del }}, \theta\right)^{\oplus m}$.

Proof By the spectral gap assumption, the family of spectral projections $\left\{p_{\Delta}\left(H_{\mathcal{L}}\right)\right\}_{\mathcal{L} \in \Omega_{\Lambda}}$ give an element $p_{\Delta}(h) \in M_{n}(\mathcal{A})$. As such, we can apply Theorem 5.15 which gives the faster than polynomially decaying tight frame or orthonormal basis of the localisation $\pi_{\mathcal{L}}\left(p_{\Delta}\right) \mathfrak{h}_{\mathcal{L}}^{\oplus n}=p_{\Delta}\left(H_{\mathcal{L}}\right) L^{2}\left(\mathbb{R}^{d}, \mathbb{C}^{n}\right)$.

Remarks 5.23 (i) The existence of an isomorphism $p_{\Delta} L^{2}\left(\mathcal{F}_{\Omega_{0}}, \theta\right)_{\mathcal{G}_{\text {Del }}}^{\oplus m} \cong C_{r}^{*}\left(\mathcal{G}_{\text {Del }}, \theta\right)^{\oplus m}$ is a $K$-theoretic statement and implies that $\left[p_{\Delta}\right]=m[1] \in K_{0}\left(C_{r}^{*}\left(\mathcal{G}_{\mathrm{Del}}, \theta\right)\right)$.

(ii) If we take a deformation of the magnetic field $\left\{\theta_{t}\right\}_{t \in[0,1]}$ such that the $\mathbb{R}$-valued 2-cocycle $\omega_{t}(x, y)=\left\langle x, \theta_{t} y\right\rangle$ is continuous in $t$, we obtain a homotopy of 2cocycles in the sense of Remark 5.16. Therefore, Theorem 5.22 is stable under 
deformations of the magnetic field provided that the spectral gap remains open throughout the deformation.

\subsection{Obstruction to Localised Wannier Basis by (Noncommutative) Chern Numbers}

Let us briefly recall the periodic setting. If the atomic potential $V_{\Lambda}=\sum_{p \in \Lambda} v(\cdot-p)$ is such that $\Lambda$ is a periodic and co-compact group $G$, then $H_{\Lambda}$ is affiliated to the algebra

$$
C_{r}^{*}\left(\Omega_{\Lambda} \rtimes \mathbb{R}^{d}, \theta\right) \cong C_{r}^{*}\left(\left(\mathbb{R}^{d} / G\right) \rtimes \mathbb{R}^{d}, \theta\right) \cong C_{r}^{*}(G, \theta) \otimes \mathbb{K}
$$

In the case $G=\mathbb{Z}^{d}$, the the non-triviality of finitely generated and projective $C_{r}^{*}\left(\mathbb{Z}^{d}, \theta\right)$-modules with $\theta$ rational can be examined by studying the Chern classes of the spectral subspaces of the Hamiltonian viewed as a complex vector bundle over the Brillouin torus, $\widehat{\mathbb{Z}}^{d}$. In the aperiodic setting, we can use tools from noncommutative geometry to carry out an analogous argument. Indeed, noncommutative Chern numbers for Hamiltonians affiliated to $C(\Omega) \rtimes_{\theta} \mathbb{R}^{d}$ and $C_{r}^{*}\left(\mathcal{G}_{\text {Del }}, \theta\right)$ have already been studied [11-13].

Throughout this section, we will regularly take advantage of the equivalence between the continuous hull $\Omega_{\Lambda} \rtimes \mathbb{R}^{d}$ and $\mathcal{G}_{\text {Del }}$, which gives an isomorphism $K_{0}\left(C_{r}^{*}\left(\Omega_{\Lambda} \rtimes \mathbb{R}^{d}, \theta\right)\right) \cong K_{0}\left(C_{r}^{*}\left(\mathcal{G}_{\text {Del }}, \theta\right)\right)$.

We first recall the top degree noncommutative Chern numbers for aperiodic or disordered magnetic Schrödinger Hamiltonians with a spectral gap. To do this, we recall the trace per unit volume on $L^{2}\left(\mathbb{R}^{d}\right)$. Let $\Lambda_{j}$ be an increasing sequence of sets that converge to $\mathbb{R}^{d}$ in an appropriate sense, e.g. $\Lambda_{j}=[-j, j]^{d}$. Then for any $a \in \mathcal{B}\left(L^{2}\left(\mathbb{R}^{d}\right)\right)$,

$$
\operatorname{Tr}_{\operatorname{Vol}}(a)=\lim _{j \rightarrow \infty} \frac{1}{\operatorname{Vol}\left(\Lambda_{j}\right)} \operatorname{Tr}\left(\Pi_{\Lambda_{j}} a\right), \quad \Pi_{\Lambda_{j}}: L^{2}\left(\mathbb{R}^{d}\right) \rightarrow L^{2}\left(\Lambda_{j}\right) .
$$

Proposition 5.24 [13] Fix a probability measure $\mathbf{P}$ on $\Omega_{\Lambda}$ that is invariant and ergodic under the $\mathbb{R}^{d}$-action and let $S_{d}$ denote the permutation group of $\{1, \ldots, d\}$. If $d>0$ is even and $p=p^{*}=p^{2} \in M_{n}(\mathcal{A})$, then for almost all $\mathcal{L} \in \Omega_{\Lambda}$ the functional

$$
C_{d}(p)=\frac{(-2 \pi i)^{d / 2}}{(d / 2) !} \sum_{\tau \in S_{d}}(-1)^{\tau}\left(\operatorname{Tr}_{\mathbb{C}^{n}} \otimes \operatorname{Tr}_{\mathrm{Vol}}\right)\left(\pi_{\mathcal{L}}(p) \prod_{j=1}^{d}\left[X_{\tau(j)}, \pi_{\mathcal{L}}(p)\right]\right)
$$

is integer valued and almost surely constant in $\Omega_{\Lambda}$.

The number $C_{d}$ almost surely defines a homomorphism $K_{0}\left(C_{r}^{*}\left(\Omega_{\Lambda} \rtimes \mathbb{R}^{d}, \theta\right)\right) \rightarrow \mathbb{Z}$, which we can also consider as a homomorphism $K_{0}\left(C_{r}^{*}\left(\mathcal{G}_{\text {Del }}, \theta\right)\right) \rightarrow \mathbb{Z}$. In particular $C_{d}(p)=C_{d}\left(p^{\prime}\right)$ if $[p]=\left[p^{\prime}\right] \in K_{0}\left(C_{r}^{*}\left(\mathcal{G}_{\text {Del }}, \theta\right)\right)$ and $C_{d}(p)=0$ if $[p]=m[1] \in$ $K_{0}\left(C_{r}^{*}\left(\mathcal{G}_{\text {Del }}, \theta\right)\right)$.

For systems with $d \geq 3$, we may also wish to consider lower-dimensional invariants. These invariants are not integer-valued in general, but can still be used to study the topology of gapped spectral projections. We fix a probability measure $\mathbf{P}$ on $\Omega_{\Lambda}$ that 
is invariant and under the $\mathbb{R}^{d}$-action. Then we recall the noncommutative calculus for $\mathcal{A} \subset C_{r}^{*}\left(\Omega_{\Lambda} \rtimes \mathbb{R}^{d}, \theta\right)$,

$$
\mathcal{T}(f)=\int_{\Omega_{\Lambda}} f(\mathcal{L}, 0) \mathrm{d} \mathbf{P}, \quad\left(\partial_{j} f\right)(\mathcal{L}, x)=x_{j} f(\mathcal{L}, x), \quad f \in \mathcal{A}, \quad j \in\{1, \ldots, d\} .
$$

Proposition 5.25 [13] Let $p=p^{*}=p^{2} \in M_{n}(\mathcal{A})$ and $\mathbf{P}$ a probability measure on $\Omega_{\Lambda}$ that is invariant and under the $\mathbb{R}^{d}$-action. Then for any $k \leq d$ an even integer, the functional

$$
C_{k}(p)=\frac{(-2 \pi i)^{k / 2}}{(k / 2) !} \sum_{\tau \in S_{k}}(-1)^{\tau}\left(\operatorname{Tr}_{\mathbb{C}^{n}} \otimes \mathcal{T}\right)\left(p \prod_{j=1}^{k} \partial_{\tau(j)} p\right)
$$

defines a homomorphism $K_{0}\left(C_{r}^{*}\left(\Omega_{\Lambda} \rtimes \mathbb{R}^{d}, \theta\right)\right) \rightarrow \mathbb{R}$. If $\mathbf{P}$ is ergodic under the $\mathbb{R}^{d}$ action and $k=d$, then $C_{k}(p)=C_{d}(p) \in \mathbb{Z}$ from Eq. (10) almost surely.

We again note that if $[p]=m[1] \in K_{0}\left(C_{r}^{*}\left(\mathcal{G}_{\text {Del }}, \theta\right)\right)$, then $C_{k}(p)=0$ for any $k \geq 2$. Combining our results on the noncommuative Chern numbers with Theorem 5.15 and the weak localisation dichotomy (Proposition 5.17), we have the following.

Corollary 5.26 Let $p=p^{*}=p^{2} \in M_{n}(\mathcal{A})$ and $\mathbf{P}$ a probability measure on $\Omega_{\Lambda}$ that is invariant under the $\mathbb{R}^{d}$-action. If $C_{k}(p)$ from $E q$. (11) is non-zero for some $k \geq 2$, then for any $\mathcal{L} \in \Omega_{0}$ there can not be Wannier basis of $\pi_{\mathcal{L}}(p) L^{2}\left(\mathbb{R}^{d}, \mathbb{C}^{n}\right)$ constructed from magnetic translations in $\mathcal{L}$ of elements $\left\{w_{j}\right\}_{j=1}^{m} \subset p \mathcal{S}_{1}\left(\mathcal{F}_{\Omega_{0}}, \theta\right)_{\mathcal{B}_{1}}^{\oplus n}$ such that for any $y \in \mathcal{L}$

$$
\sum_{j=1}^{m} \int_{\mathbb{R}^{d}}\left(1+|x-y|^{2}\right)\left|\left(U_{y} w_{j}^{\mathcal{L}}\right)(x)\right|^{2} \mathrm{~d} x<\infty
$$

\subsection{Deformation of the Delone Atomic Potential}

We would like to consider the stability of our results on aperiodic Schrödinger operators $H_{\Lambda}$ when the Delone set $\Lambda$ is deformed (e.g. from an aperiodic set to a periodic lattice). Deforming a Delone set $\Lambda$ will change the crossed product groupoid and the $K$-theory may change substantially as the Delone set changes. However, we will show the pairings in cyclic cohomology considered in Sect. 5.5 are unaffected by such deformations.

Lemma 5.27 Let $v \in C_{c}\left(\mathbb{R}^{d}\right)$ be a continuous atomic potential with compact support. If $\left\{\Lambda_{t}\right\}_{t \in[0,1]}$ is continuous path of $(r, R)$-Delone sets in $\operatorname{Del}_{(r, R)}$, then the path of Schrödinger operators $\left\{H_{\Lambda_{t}}\right\}_{t \in[0,1]}$ is norm-continuous in the resolvent topology. 
Proof It is straightforward to see that for any $v \in C_{c}\left(\mathbb{R}^{d}\right), v \in L_{K, r}^{1}\left(\mathbb{R}^{d}\right)$ and the function $v_{\Lambda}(x)=\sum_{p \in \Lambda} v(p-x)$ is real-valued, measurable and essentially bounded for any $(r, R)$-Delone set $\Lambda$. Because $\operatorname{Dom}\left(H_{\Lambda}\right)$ is constant for any $\Lambda \in \operatorname{Del}_{(r, R)}$, we can use the resolvent identity to bound

$$
\begin{aligned}
\left\|\left(z-H_{\Lambda_{1}}\right)^{-1}-\left(z-H_{\Lambda_{2}}\right)^{-1}\right\| & =\left\|\left(z-H_{\Lambda_{1}}\right)^{-1}\left(H_{\Lambda_{1}}-H_{\Lambda_{2}}\right)\left(z-H_{\Lambda_{2}}\right)^{-1}\right\| \\
& \leq \text { ess. } \sup \left|v_{\Lambda_{1}}-v_{\Lambda_{2}}\right|\left\|\left(z-H_{\Lambda_{1}}\right)^{-1}\right\|\left\|\left(z-H_{\Lambda_{2}}\right)^{-1}\right\|
\end{aligned}
$$

for any $z \in \mathbb{C} \backslash \mathbb{R}$. The result will therefore follow if we can show that the essential supremum is controlled by the topology on $\operatorname{Del}_{(r, R)}$. Suppose that $\operatorname{supp}(v) \subset B(0 ; M)$ for some $M>0$. Recalling the topology of $\operatorname{Del}_{(r, R)}$ (Proposition 5.2) with $d_{H}$ the Hausdorff metric, we take $x \in \mathbb{R}^{d}$ and suppose that $d_{H}\left(\Lambda_{1}-x \cap B(0 ; M), \Lambda_{2}-x \cap\right.$ $B(0 ; M)) \leq \delta<r / 2$. Taking $\delta$ small enough, we can ensure that $\mid \Lambda_{1}-x \cap B(0 ; M+$ $r / 2)|=| \Lambda_{2}-x \cap B(0 ; M+r / 2) \mid$ and, furthermore, we can decompose the sets $\Lambda_{1}-x \cap B(0 ; M+r / 2)$ and $\Lambda_{2}-x \cap B(0 ; M+r / 2)$ as pairs $(p, q) \in \Lambda_{M}:=$ $\Lambda_{1} \cap B(x ; M+r / 2) \times \Lambda_{2} \cap B(x ; M+r / 2)$ such that $\|p-q\| \leq \delta$. We can therefore estimate

$$
\begin{aligned}
\left|\left(v_{\Lambda_{1}}-v_{\Lambda_{2}}\right)(x)\right| & =\left|\sum_{p \in \Lambda_{1}} v(p-x)-\sum_{q \in \Lambda_{2}} v(q-x)\right| \\
& =\left|\sum_{p \in \Lambda_{1} \cap B(x ; M)} v(p-x)-\sum_{q \in \Lambda_{2} \cap B(x ; M)} v(q-x)\right| \\
& =\left|\sum_{\substack{(p, q) \in \Lambda_{M} \\
\|p-q\| \leq \delta}} v(p-x)-v(q-x)\right| \\
& \leq \sum_{\substack{(p, q) \in \Lambda_{M} \\
\|p-q\| \leq \delta}}|v(p-x)-v(q-x)| .
\end{aligned}
$$

By continuity of $v$, given any $\varepsilon>0$ we can choose a small enough $\delta$ so that $\mid v(p-$ $x)-v(q-x) \mid<\frac{\varepsilon}{\left|\Lambda_{1} \cap B(x ; M+r / 2)\right|}$ for all $\|p-q\| \leq \delta$. Hence $\left|\left(v_{\Lambda_{1}}-v_{\Lambda_{2}}\right)(x)\right|<\varepsilon$ and the essential supremum is also bounded by $\varepsilon$.

Definition 5.28 Let $\left\{\Lambda_{t}\right\}_{t \in[0,1]}$ be a continuous path in $\operatorname{Del}_{(r, R)}$. We say that $\left\{H_{\Lambda_{t}}\right\}_{t \in[0,1]}$ is a gapped path if there exists a bounded interval $\Delta \subset \mathbb{R}$ such that for all $t \in[0,1]$ and $\mathcal{L}_{t} \in \Omega_{\Lambda_{t}}, \Delta \cap \sigma\left(H_{\mathcal{L}_{t}}\right)$ is non-empty and $\Delta$ is separated from the rest of the spectrum of $H_{\mathcal{L}_{t}}$ by a positive distance.

The conditions to obtain a gapped path are quite strong, but if satisfied give a path of operators $\left\{h_{t}\right\}_{t \in[0,1]}$ such that $h_{t}$ is affiliated to $C_{r}^{*}\left(\Omega_{\Lambda_{t}} \rtimes \mathbb{R}^{d}, \theta\right)$ and $p_{t}=\chi_{\Delta}\left(h_{t}\right) \in$ $\mathcal{A}_{t} \subset C_{r}^{*}\left(\Omega_{\Lambda_{t}} \rtimes \mathbb{R}^{d}, \theta\right)$, a dense pre- $C^{*}$-algebra.

Proposition 5.29 Let $\left\{\Lambda_{t}\right\}_{t \in[0,1]}$ be a continuous path in $\operatorname{Del}_{(r, R)}$ and fix an atomic potential $v \in C_{c}\left(\mathbb{R}^{d}\right)$. Suppose that $\left\{H_{\Lambda_{t}}\right\}_{t \in[0,1]}$ is a gapped path with isolated spectral region $\Delta \subset \mathbb{R}$. Then for $p_{t}=\left\{\chi_{\Delta}\left(H_{\mathcal{L}_{t}}\right)\right\}_{\mathcal{L}_{t} \in \Omega_{\Lambda_{t}}} \in \mathcal{A}_{t}$ and any even integer $k \leq d$, 
the function

$$
[0,1] \ni t \mapsto C_{k}\left(p_{t}\right)=\frac{(-2 \pi i)^{k / 2}}{(k / 2) !} \sum_{\tau \in S_{k}}(-1)^{\tau}\left(\operatorname{Tr}_{\mathbb{C}^{n}} \otimes \mathcal{T}\right)\left(p_{t} \prod_{j=1}^{k} \partial_{\tau(j)} p_{t}\right) \in \mathbb{R}
$$

is continuous, where $C_{k}(p)$ is the weak Chern number from Eq. (11).

Proof The assumption on the spectral gap implies that $C_{k}\left(p_{t}\right)$ is well-defined for all $t$. Because we have a uniform isolated spectral region $\Delta$, we can write for all $t \in[0,1]$,

$$
p_{t}=\frac{1}{2 \pi i} \oint_{\mathcal{C}}\left(z-h_{t}\right)^{-1} \mathrm{~d} z, \quad h_{t}=\left\{\left(z-H_{\mathcal{L}_{t}}\right)^{-1}\right\}_{\mathcal{L}_{t} \in \Omega_{\Lambda_{t}}}
$$

with $\mathcal{C}$ a contour enclosing $\Delta$ and not intersecting any other part of the spectrum. If $\left\{\Lambda_{t}\right\}_{t \in[0,1]}$ is a continuous path in $\operatorname{Del}_{(r, R)}$, then there is a corresponding continuous path $\left\{\mathcal{L}_{t}\right\}_{t \in[0,1]}$ with $\mathcal{L}_{t}$ in the orbit space of $\Lambda_{t}$. Because $t \mapsto\left(z-H_{\mathcal{L}_{t}}\right)^{-1}$ is normcontinuous by Lemma 5.27, so is $t \mapsto\left\|\left(z-h_{t}\right)^{-1}\right\|$. By the integral formula for the spectral projections, $t \mapsto\left\|p_{t}\right\|$ is continuous. Because the functional $C_{k}$ induces a weaker topology than the norm topology, $t \mapsto C_{k}\left(p_{t}\right)$ is also continuous.

Continuity of the function $[0,1] \ni t \mapsto\left\|\left(z-h_{t}\right)^{-1}\right\| \in \mathbb{R}$ for all $z$ in the resolvent set implies that the spectral edges of $\sigma\left(h_{t}\right)$ are continuous away from gap closing points, see [3].

Continuity of the Chern numbers under deformations of Delone sets means that if the range of the pairing is quantised, then it is constant under deformations.

Corollary 5.30 Let $\left\{\Lambda_{t}\right\}_{t \in[0,1]}$ be a continuous path in $\operatorname{Del}_{(r, R)}$ and fix an atomic potential $v \in C_{c}\left(\mathbb{R}^{d}\right)$. Suppose that $\left\{H_{\Lambda_{t}}\right\}_{t \in[0,1]}$ is a gapped path with isolated spectral region $\Delta \subset \mathbb{R}$. Let $\mathbf{P}_{0}$ be an invariant and ergodic probability measure on $\Omega_{\Lambda_{0}}$. Then for almost all $\mathcal{L}_{0} \in \Omega_{\Lambda_{0}}$,

$$
\begin{aligned}
C_{d}\left(p_{t}\right) & =C_{d}\left(p_{\mathcal{L}_{0}}\right) \\
& :=\frac{(-2 \pi i)^{d / 2}}{(d / 2) !} \sum_{\tau \in S_{d}}(-1)^{\tau}\left(\operatorname{Tr}_{\mathbb{C}^{n}} \otimes \operatorname{Tr}_{\mathrm{Vol}}\right)\left(\chi_{\Delta}\left(H_{\mathcal{L}_{0}}\right) \prod_{j=1}^{d}\left[X_{\tau(j)}, \chi_{\Delta}\left(H_{\mathcal{L}_{0}}\right)\right]\right)
\end{aligned}
$$

is integer valued and constant for all $t \in[0,1]$.

Corollaries 5.30 and 5.26 then give us the following stability result on delocalised Wannier bases.

Corollary 5.31 (Stability of delocalised Wannier basis under atomic deformations) Let $\Lambda_{0}$ be an $(r, R)$-Delone set and consider $H_{\Lambda_{0}}$ with atomic potential $v \in C_{c}\left(\mathbb{R}^{d}\right)$. Fix an invariant and ergodic probability measure on $\Omega_{\Lambda_{0}}$ and suppose that $C_{d}\left(p_{\mathcal{L}_{0}}\right) \neq 0$ for almost all $\mathcal{L}_{0} \in \Omega_{\Lambda_{0}}$. Then for any gapped path $\left\{H_{\Lambda_{t}}\right\}_{t \in[0,1]}$ with isolated spectral region $\Delta \subset \mathbb{R}$ and any $\mathcal{L}_{t}$ in the transversal $\Omega_{0, t}$, there can not be a faster than polynomially decaying Wannier basis of $\chi_{\Delta}\left(H_{\mathcal{L}_{t}}\right) L^{2}\left(\mathbb{R}^{d}, \mathbb{C}^{n}\right)$ built from magnetic translates in $\mathcal{L}_{t}$. 
We again note that by Proposition 5.17 the non-existence of a faster than polynomially decaying Wannier basis also implies that the weaker localisation bound of Eq. (8) diverges,

$$
\sum_{j=1}^{m} \int_{\mathbb{R}^{d}}\left(1+|x|^{2}\right)\left|w_{j}^{\mathcal{L}}(x)\right|^{2} \mathrm{~d} x=\infty .
$$

Acknowledgements The authors thank Franz Luef, Domenico Monaco and Guo Chuan Thiang for valuable feedback on an earlier version of this manuscript. We also thank Giovanna Marcelli, Massimo Moscolari and Gianluca Panati for sharing the results of $[29,30]$ with us. CB is supported by a JSPS Grant-in-Aid for Early-Career Scientists (No. 19K14548) and thanks the Mathematical Institute, Universiteit Leiden, for hospitality during the conference Noncommutative Geometry, Analysis, and Topological Insulators in February 2020, where this work took shape. Both authors thank the Casa Matematica Oaxaca for hospitality and support during the workshop Topological Phases of Interacting Quantum Systems in June 2019.

\section{References}

1. Austad, A., Enstad, U.: Heisenberg modules as function spaces. J. Fourier Anal. Appl. 26, 24 (2020)

2. Austad, A., Jakobsen, M.S., Luef, F.: Gabor duality theory for Morita equivalent $C^{*}$-algebras. Int. J. Math. 31(10), 2050073 (2020)

3. Beckus, S., Bellissard, J.: Continuity of the spectrum of a field of self-adjoint operators. Ann. Henri Poincaré 17(12), 3425-3442 (2016)

4. Beckus, S., Bellissard, J., De Nittis, G.: Spectral continuity for aperiodic quantum systems I. Gen. Theory J. Funct. Anal. 275(11), 2917-2977 (2018)

5. Bellissard, J.: Gap labelling theorems for Schrödinger operators. In: Waldschmidt, M., et al. (eds.) From Number Theory to Physics, Chapter 12. Springer, Berlin (1992)

6. Bellissard, J., Herrmann, D.J.L., Zarrouati, M.: Hulls of aperiodic solids and gap labelling theorems. In: Directions in Mathematical Quasicrystals. Volume 13 of CIRM Monograph Series, pp. 207-259 (2000)

7. Belmonte, F., Lein, M., Măntoiu, M.: Magnetic twisted actions on general abelian $C^{*}$-algebras. J. Oper. Theory 69(1), 33-58 (2013)

8. Benac, M.J., Massey, P.G., Stojanoff, D.: Frames of translates with prescribed fine structure in shift invariant spaces. J. Funct. Anal. 271(9), 2631-2671 (2016)

9. Blackadar, B.: $K$-Theory for Operator Algebras. Volume 5 of Mathematical Sciences Research Institute Publications. Cambridge University Press, Cambridge (1998)

10. Blackadar, B., Cuntz, J.: Differential Banach algebra norms and smooth subalgebras of $C^{*}$-algebras. J. Oper. Theory 26, 255-282 (1991)

11. Bourne, C., Mesland, B.: Index theory and topological phases of aperiodic lattices. Annales Henri Poincaré 20(6), 1969-2038 (2019)

12. Bourne, C., Prodan, E.: Non-commutative Chern numbers for generic aperiodic discrete systems. J. Phys. A 51(23), 235202 (2018)

13. Bourne, C., Rennie, A.: Chern numbers, localisation and the bulk-edge correspondence for continuous models of topological phases. Math. Phys. Anal. Geom. 21(3), 16 (2018)

14. Christensen, O.: An Introduction to Frames and Riesz Bases. Applied and Numerical Harmonic Analysis, 2nd edn. Birkhäuser/Springer, Cham (2016)

15. Cornean, H.D., Monaco, D., Moscolari, M.: Parseval frames of exponentially localized magnetic Wannier functions. Commun. Math. Phys. 371(3), 1179-1230 (2019)

16. De Nittis, G., Lein, M.: Exponentially localized Wannier functions in periodic zero flux magnetic fields. J. Math. Phys. 52(11), 112103 (2011)

17. Gillaspy, E.: $K$-theory and homotopies of 2-cocycles on transformation groups. J. Oper. Theory $73(2)$, 465-490 (2015)

18. Gracia-Bondía, J.M., Várilly, J.C., Figueroa, H.: Elements of Noncommutative Geometry. Birkhäuser Advanced Texts Basler Lehrbücher. Birkhäuser, Boston (2001) 
19. Gröchenig, K., Ortega-Cerdà, J., Romero, J.L.: Deformation of Gabor systems. Adv. Math. 277, 388425 (2015)

20. Han, D., Larson, D.R.: Frames, bases and group representations. Mem. Am. Math. Soc. 147(697), $\mathrm{x}+94(2000)$

21. Kellendonk, J.: The local structure of tilings and their integer group of coinvariants. Commun. Math. Phys. 187, 115-157 (1997)

22. Khoshkam, M., Skandalis, G.: Regular representation of groupoid $C^{*}$-algebras and applications to inverse semigroups. J. Reine Angew. Math. 546, 47-72 (2002)

23. Kreisel, M.: Gabor frames for quasicrystals, $K$-theory, and twisted gap labeling. J. Funct. Anal. 270, 1001-1030 (2016)

24. Kuchment, P.: Tight frames of exponentially decaying Wannier functions. J. Phys. A 42(2), 025203 (2009)

25. Lu, J., Stubbs, K.: Algebraic localization implies exponential localization in non-periodic insulators. arXiv:2101.02626 (2021)

26. Ludewig, M., Thiang, G.C.: Good Wannier bases in Hilbert modules associated to topological insulators. J. Math. Phys. 61, 061902 (2020)

27. Luef, F.: Projective modules over noncommutative tori are multi-window Gabor frames for modulation spaces. J. Funct. Anal. 257(6), 1921-1946 (2009)

28. Luef, F.: The Balian-Low theorem and noncommutative tori. Expos. Math. 36(2), 221-227 (2018)

29. Marcelli, G., Monaco, D., Moscolari, M., Panati, G.: The Haldane model and its localization dichotomy. Rend. Mat. Appl. (7) 39(2), 307-327 (2018). arXiv:1909.03298

30. Marcelli, G., Moscolari, M., Panati, G.: Localization implies Chern triviality in non-periodic insulators. arXiv:2012.14407 (2020)

31. Matusiak, E.: Gabor frames for model sets. J. Fourier Anal. Appl. 25(5), 2570-2607 (2019)

32. Monaco, D., Panati, G., Pisante, A., Teufel, S.: Optimal decay of Wannier functions in Chern and quantum Hall insulators. Commun. Math. Phys. 359(1), 61-100 (2018)

33. Moutuou, E. M., Tu, J.-L.: Equivalence of fell systems and their reduced groupoid $C^{*}$-algebras. arXiv:1101.1235 (2011)

34. Muhly, P.S., Renault, J., Williams, D.P.: Equivalence and isomorphism for groupoid $C^{*}$-algebras. J. Oper. Theory 17, 3-22 (1987)

35. Raeburn, I., Williams, D.: Morita Equivalence and Continuous-Trace $C^{*}$-Algebras. Mathematical Surveys and Monographs, vol. 60. American Mathematical Society, Providence, RI, xiv+327 (1998)

36. Renault, J.: A Groupoid Approach to $C^{*}$-Algebras. Lecture Notes in Mathematics, vol. 793. Springer, Berlin (1980)

37. Schweitzer, L.B.: A short proof that $M_{n}(A)$ is local if $A$ is local and Fréchet. Int. J. Math. 3(4), 581-589 (1992)

38. Sims, A., Williams, D.P.: Renault equivalence Theorem for reduced groupoid $C^{*}$-algebras. J. Oper. Theory 68(1), 223-239 (2012)

39. Sims, A., Williams, D.P.: An equivalence theorem for reduced Fell bundle $C^{*}$-algebras. N. Y. J. Math. 19, 159-178 (2013)

40. Stubbs, K., Watson, A. B., Lu, J.: Existence and computation of generalized Wannier functions for non-periodic systems in two dimensions and higher. arXiv:2003.06676 (2020)

41. Stubbs, K., Watson, A.B., Lu, J.: The iterated projection position algorithm for constructing exponentially localized generalized Wannier functions for periodic and non-periodic insulators in two dimensions and higher. Phys. Rev. B 103, 075125 (2021)

Publisher's Note Springer Nature remains neutral with regard to jurisdictional claims in published maps and institutional affiliations. 